NBER WORKING PAPER SERIES

\title{
CONSUMPTION COMMITMENTS AND HABIT FORMATION
}

\author{
Raj Chetty \\ Adam Szeidl \\ Working Paper 10970 \\ http://www.nber.org/papers/w10970 \\ NATIONAL BUREAU OF ECONOMIC RESEARCH \\ 1050 Massachusetts Avenue \\ Cambridge, MA 02138 \\ December 2004
}

Previously circulated as "Consumption Commitments: Neoclassical Foundations for Habit Formation." For helpful comments we thank John Campbell, Gary Chamberlain, Tom Davido,, Drew Fudenberg, Elhanan Helpman, Miklos Koren, Hanno Lustig, Todd Sinai, Jeremy Stein, and Moto Yogo. For funding, Chetty and Szeidl thank the National Science Foundation (Grant SES 0522073), Szeidl thanks the Social Science Research Council, the Institute for Humane Studies, and the European Research Council under the European Unions Seventh Framework Program (FP7/2007-2013), ERC grant agreement number 283484. The views expressed herein are those of the author and do not necessarily reflect the views of the National Bureau of Economic Research.

NBER working papers are circulated for discussion and comment purposes. They have not been peerreviewed or been subject to the review by the NBER Board of Directors that accompanies official NBER publications.

(C) 2004 by Raj Chetty and Adam Szeidl. All rights reserved. Short sections of text, not to exceed two paragraphs, may be quoted without explicit permission provided that full credit, including (C notice, is given to the source. 
Consumption Commitments and Habit Formation

Raj Chetty and Adam Szeidl

NBER Working Paper No. 10970

December 2004, Revised September 2015

JEL No. D8,E21,G11,G12

\begin{abstract}
$\underline{\text { ABSTRACT }}$
We analyze the implications of household-level adjustment costs for the dynamics of aggregate consumption. We show that an economy in which agents have "consumption commitments" is approximately equivalent to a habit formation model in which the habit stock is a weighted average of past consumption if idiosyncratic risk is large relative to aggregate risk. Consumption commitments can thus explain the empirical regularity that consumption is excessively sensitive and excessively smooth, findings that are typically attributed to habit formation. Unlike habit formation and other theories, but consistent with empirical evidence, the consumption commitments model also predicts that excess sensitivity and smoothness vanish for large shocks. These results suggest that behavior previously attributed to habit formation may be better explained by adjustment costs. We develop additional testable predictions to further distinguish the commitment and habit models and show that the two models have different welfare implications.
\end{abstract}

\author{
Raj Chetty \\ Department of Economics \\ Harvard University \\ 1805 Cambridge St. \\ Cambridge, MA 02138 \\ and NBER \\ chetty@fas.harvard.edu \\ Adam Szeidl \\ Department of Economics \\ Central European University \\ Nador u. 11 \\ Budapest, Hungary \\ and NBER \\ szeidla@ceu.hu
}




\section{Introduction}

Many households have "consumption commitments" such as housing that are costly to adjust in response to fluctuations in income. Chetty and Szeidl (2007) document that more than 50\% of the average U.S. household's budget remains fixed when the household faces moderate income shocks such as unemployment. Olney (1999) gives historical evidence on the importance of households' installment finance commitments during the Great Depression. Such consumption commitments can amplify the welfare costs of shocks because - for shocks that are not large enough to induce a change in commitments - households are forced to concentrate all reductions in wealth on changes in adjustable (e.g., food) consumption. Through this mechanism, consumption commitments can help explain microeconomic evidence in domains ranging from wage rigidities (Postlewaite, Samuelson and Silverman 2008) to added-worker effects (Chetty and Szeidl 2007), housing choices of couples (Shore and Sinai 2009), and portfolio choice (Chetty and Szeidl 2014).

In this note, we show that household-level consumption commitments also have important implications at the macroeconomic level, especially for the dynamics of aggregate consumption. We show that when idiosyncratic risk is large relative to aggregate risk, nonlinear dynamics due to commitments at the household level aggregate into approximately linear dynamics for larger groups, producing patterns that are approximately identical to representative-agent habit formation. ${ }^{1}$ In particular, commitments can explain the key facts - often attributed to habit formation - that consumption exhibits excess sensitivity and excess smoothness. But the commitments model also explains empirical regularities that are not consistent with standard habit formation models. For instance, it predicts that excess sensitivity and smoothness vanish for large shocks, providing foundations for an empirical phenomenon termed the "magnitude hypothesis" (Japelli and Pistaferri 2010). Hence, our results suggest that some of the behavior previously attributed to habit formation may be due to adjustment costs in consumption. The distinction between the two models matters because they generate different comparative statics and yield different welfare implications.

We begin our analysis in Section 2 with a household-level model in which changing the consumption of certain goods is costly. These costs could reflect either transaction costs or mental costs such as the effort required for changing plans (Grossman and Laroque 1990, Chetty and

\footnotetext{
${ }^{1}$ Beginning with Ryder and Heal (1973), models in which habit is an average of past consumption are widely used in economics. Sundaresan (1989), Constantinides (1990), Campbell and Cochrane (1999) and Boldrin, Christiano and Fisher (2001) use variants of this model in macro-finance, while Carroll, Overland and Weil (2000), Fuhrer (2000), Christiano, Eichenbaum, and Evans (2003) and a literature building on this work uses variants in macroeconomics and monetary policy.
} 
Szeidl 2007). We show that in an economy populated by many such agents, aggregate dynamics can be represented by the preferences of a representative agent whose utility function involves a state variable corresponding to aggregate commitments. ${ }^{2}$ This state variable is endogenous: each household chooses commitments to maximize expected utility, and hence aggregate commitments are shaped by the expectations agents hold on the dates on which they update.

In Section 3, we characterize the aggregate dynamics of consumption commitments. Our main result is a precise characterization showing that when the ratio of idiosyncratic to aggregate consumption risk is large, aggregate commitments are well approximated by a (linear) weighted average of past consumption with fixed weights. As a result, the commitments economy is closely approximated by a representative-agent habit model in which the habit stock is a weighted average of past consumption. ${ }^{3} \quad$ To understand this result, note that the impulse response to aggregate shocks in the commitments model depends on the distribution of agents in the inaction region for commitment consumption. Aggregate shocks perturb this distribution, while idiosyncratic shocks push it back towards its steady state. When idiosyncratic risk is large, the second effect dominates, and hence on most dates the distribution remains close to its steady state. Thus impulse-responses are approximately state-independent, which in turn can be generated in a habit model with fixed weights. Since in practice idiosyncratic risk is much larger than economy-wide risk (e.g., Deaton, 1991, Carroll, Hall, and Zeldes 1992), we interpret this result as showing that consumption commitments and habit formation generate similar aggregate consumption dynamics in a typical environment.

While the commitments model matches the predictions of habit models in a commonly-studied domain, it yields new predictions in other settings. In Section 4, we illustrate the similarities and differences between the two models using three applications. We first consider the consumption response to income shocks. Two well-documented empirical regularities are that consumption does not respond fully to contemporaneous shocks ("excess smoothness," Deaton 1987) and that anticipated changes affect current consumption ("excess sensitivity," Flavin 1981). Fuhrer (2000) argues that both of these facts can be explained by a habit formation model in which habit responds sluggishly to shocks, which is one reason why habit models have been influential in macroeconomics. Our equivalence result implies that the commitments model also produces sluggish responses in most

\footnotetext{
${ }^{2}$ In our economy the relative price of commitment and adjustable consumption is exogenous and fixed. We discuss both general equilibrium and partial equilibrium interpretations of this assumption in Section 2.2.

${ }^{3}$ Our characterization is analytical. Previous studies of aggregate consumption with adjustment costs use numerical techniques (Marshall and Parekh 1999), or time-dependent adjustment (Lynch 1996, Gabaix and Laibson 2001, Reis 2006). These studies focus on a model with a single illiquid good, as in Grossman and Laroque (1990).
} 
periods and therefore also explains excess sensitivity and smoothness.

However, a key prediction of the commitments model - but not the habit model - is that the excess sensitivity of consumption vanishes for large shocks. When such shocks occur, households adjust their commitments and thus behave more in line with the permanent income model. This prediction helps explain a large body of micro evidence about consumption responses to shocks termed the "magnitude hypothesis" by Japelli and Pistaferri (2010). For example, Hsieh (2003) finds that Alaskan households' consumption is excessively sensitive to tax refunds (a small income change), but not to payments from the Alaska Permanent Fund (a large income change). Similarly, Parker (1999), Souleles (1999) and Souleles (2002) find excess sensitivity to small income changes associated with tax and social security payments, but Browning and Collado (2001) and Souleles (2000) find no excess sensitivity to large changes in disposable income coming from bonus salary payments and college tuition. Such facts are difficult to explain with standard habit models, in which the impulse response to income shocks does not depend on shock size. They can, however, be explained by the commitments model, suggesting that a significant part of consumption behavior attributed to habits in preferences may be due to adjustment costs in consumption.

In our second application, we explore how consumption dynamics are affected by changes in the environment. Because commitments are chosen by the consumer, they respond endogenously to such changes. In contrast, the parameters determining reduced-form habit are exogenous and do not vary with the environment. We show that reductions in risk or in expected growth increase sluggishness of consumption in the commitment economy because they reduce the frequency of adjustment. This result yields a new prediction about excess sensitivity: consumption should respond more quickly to shocks in high-growth and high-risk environments. At the macroeconomic level, this logic suggests that recessions may be shorter lived in rapidly growing economies, in which agents reorganize their arrangements frequently. Similarly, recessions may last longer in welfare states that have large social safety nets. Evidence on these predictions would help further distinguish between the commitments and habit models.

In our final application, we turn to welfare analysis. Distinguishing between the commitments and habit models is especially important because the two models have different welfare and policy implications. We first note that the commitment model has a natural welfare measure based on expected utility. In the habit model, such a measure is not immediately available. Prior work (e.g., Ljungquist and Uhlig 2000, Ljungquist and Uhlig 2009) has assumed that the welfare of agents with habit preferences is fully determined by surplus consumption, without including the 
habit stock. We follow this approach and compare the welfare cost of a shock-measured by the willingness to pay to avoid it - in the two models. We find that the welfare cost of large shocks is smaller in the commitments model than in the habit model because agents can abandon their commitments but not their habits in extreme events. This result suggests that the optimal size of social insurance programs that insure large shocks such as disability or job displacement may be smaller than predicted by analyses using habit models such as Ljungquist and Uhlig (2000). We also find that reducing idiosyncratic risk - e.g., by expanding social insurance programs - can increase the welfare cost of aggregate shocks by slowing the rate of adjustment.

Our results build on two strands of prior research. First, several papers have pointed out the qualitative similarity between the commitment and habit models. Dybvig (1995) examines ratcheting consumption demand under extreme habit persistence and motivates these preferences by pre-commitment in consumption. Flavin and Nakagawa (2008) study asset pricing in a twogood adjustment cost model and note the similarity to habit. Fratantoni (2001) and Postlewaite, Samuelson and Silverman (2008) also study two-good models and note this similarity in other contexts. We contribute to this literature by analyzing aggregate dynamics, presenting formal conditions under which commitments and habit formation are similar, and deriving new behavioral and welfare predictions that distinguish the two models (summarized in Section 5).

Second, our results also build on a literature on industry dynamics, including Bertola and Caballero (1990), Caballero (1993), and Caballero and Engel (1993, 1999). Our main innovation relative to this literature is to develop a theory of state-dependent impulse responses, which we then use to derive an analytical characterization of aggregate dynamics. Our habit equivalence result is also related to Khan and Thomas (2008), who establish approximate linearity in a production setting with endogenous prices computationally. We establish approximate linearity - emerging through a different mechanism - in a consumption setting with exogenous prices analytically.

\section{A Model of Consumption Commitments}

In this section, we present our model, characterize household behavior, and show the existence of a representative consumer in our setting. We present a map of all proofs in the Appendix, and full technical details in a Supplementary Appendix. 


\section{$2.1 \quad$ Setup}

We study a continuous-time economy with a unit mass of consumers. We index agents by $i \in[0,1]$, but suppress the index in notation for simplicity when it does not cause confusion. Each agent maximizes expected lifetime utility given by

$$
\mathrm{E} \int_{0}^{\infty} e^{-\rho t}\left(\kappa \frac{a_{t}^{1-\gamma}}{1-\gamma}+\frac{x_{t}^{1-\gamma}}{1-\gamma}\right) \mathrm{d} t
$$

where $\rho$ is the discount rate. Each agent consumes two goods: $a_{t}$ and $x_{t}$ measure the service flows from adjustable (e.g., food) and commitment (e.g., housing) consumption, and $\kappa$ measures the relative preference for adjustables. Adjusting commitment consumption $x_{t}$ involves a fixed monetary cost, which may depend both on the pre-existing and new service flow from commitment consumption. Formally, denoting $x_{t-}=\lim \sup _{s / t} x_{s}$, if on date $t$ the agent sets $x_{t} \neq x_{t-}$, he must pay a monetary cost of $\lambda_{1} x_{t-}+\lambda_{2} x_{t}$ where $\lambda_{1}, \lambda_{2} \geq 0$ and at least one of them is positive. ${ }^{4}$

We are interested in characterizing how individual heterogeneity translates into aggregate dynamics in the presence of consumption commitments. We therefore study an economy in which agents are exposed to both idiosyncratic and aggregate risk. We introduce these risks by assuming that agents have access to a variety of financial assets. The return processes of all assets are technologically determined and exogenous, and all returns are paid out in the adjustable good. Each agent can invest in a bond with a constant instantaneous riskfree return $r$, so that the face value of the bond evolves as

$$
d B_{t} / B_{t}=r d t
$$

We also allow two types of risky investments, both with i.i.d. returns. The source of aggregate risk is the stock market, with instantaneous return

$$
d S_{t} / S_{t}=(r+\pi) d t+\sigma d z_{t}
$$

where $z_{t}$ is a standard Brownian motion that generates a filtration $\left\{\mathcal{F}_{t}, 0 \leq t<\infty\right\}, \pi$ is the expected excess return, and $\sigma$ is the standard deviation of asset returns. Households also face idiosyncratic risk in the form of a household-specific risky investment opportunity. This background risk can be thought of as entrepreneurial investment or labor income risk (where "investment" is

\footnotetext{
${ }^{4}$ Similar utility and adjustment cost specifications have been used by Flavin and Nakagawa (2008), Fratantoni (2001), Li (2003), and Postlewaite, Samuelson and Silverman (2008).
} 
investment in human capital). The return of household $i$ 's entrepreneurial investment is given by

$$
d S_{t}^{E, i} / S_{t}^{E, i}=\left(r+\pi_{E}\right) d t+\sigma_{E} d z_{t}^{i}
$$

where the $z^{i}$ s are standard Brownian motions uncorrelated across households. ${ }^{5}$ Each agent can invest or disinvest any amount into his private asset at any time. We ignore imperfections in financial markets: an agent can go long and short in any of the assets available to him.

We assume that the relative price of adjustable and commitment consumption services is exogenous and normalized to one. We discuss below both general equilibrium and partial equilibrium interpretations of this assumption. We also assume that the agent pays for the commitment consumption service every period (e.g., as with rental housing) ${ }^{6}$ Denoting total wealth by $w_{t}^{i}$, the wealth share invested in the stock market by $\alpha_{t}^{i}$, and the wealth share invested in the entrepreneurial asset by $\alpha_{t}^{E, i}$, the dynamic budget constraint of agent $i$ is

$$
d w_{t}^{i}=w_{t}^{i}\left[\alpha_{t}^{i} \frac{d S_{t}}{S_{t}}+\alpha_{t}^{E, i} \frac{d S_{t}^{E, i}}{S_{t}^{E, i}}+\left(1-\alpha_{t}^{i}-\alpha_{t}^{E, i}\right) \frac{d B_{t}}{B_{t}}\right]-\left(a_{t}+x_{t}\right) d t-1_{\left\{x_{t-\neq} \neq x_{t}\right\}}\left(\lambda_{1} x_{t-}+\lambda_{2} x_{t}\right) .
$$

We make the standard assumption that $\rho>(1-\gamma) r+\left[\pi^{2} /\left(2 \sigma^{2}\right)+\pi_{E}^{2} /\left(2 \sigma_{E}^{2}\right)\right](1-\gamma) / \gamma$, which ensures that with zero adjustment costs, expected consumption utility grows at a smaller rate than the discount rate in the optimum, generating finite lifetime utility.

\subsection{Discussion of Modelling Choices}

Consumption commitments. As a benchmark, we interpret the adjustment cost as the physical transaction cost inherent in changing consumption of illiquid durables such as houses, cars, or appliances, or the cost of renegotiating service contracts (Attanasio 2000, Eberly 1994, Grossman and Laroque 1990). ${ }^{7}$ However, the adjustment cost may also represent costs required to respond to new circumstances and make new choices (Browning and Collado 2001, Ergin 2003), and may arise from attention costs or computing costs (Ameriks, Caplin and Leahy, 2003, Reis, 2006).

Exogenous price of commitment good. The fixed relative price of the commitment good can

\footnotetext{
${ }^{5}$ Sun (1998) develops the mathematical foundations for working with a "large number" of independent stochastic processes, and derives exact laws of large numbers in these settings.

${ }^{6}$ Because the agent is free to borrow, this model is equivalent to one in which the agent buys and sells the capitalized service flow at price $x / r$ on every adustment date.

${ }^{7}$ More recently, several studies have examined state-dependent models with two consumption components, one freely adjustable and one that is costly to adjust (Flavin and Nakagawa 2008, Fratantoni 2003, Li 2003).
} 
be interpreted as arising from a technology that can transform adjustable into commitment and commitment into adjustable goods at a given conversion rate, after paying the adjustment cost. With this interpretation, since investment opportunities are also technologically determined, our model environment is a general equilibrium economy.

An alternative, partial equilibrium interpretation of the exogenous price assumption is that the model describes a group of people who are small from the perspective of the aggregate economy. In this interpretation "aggregate shocks" affect all members of the group but are uncorrelated with economy-wide fluctuations; and a "representative agent" represents the group, not the entire economy. This partial equilibrium interpretation is closest to the micro evidence we discuss in the context of excess sensitivity and smoothness in Section 4.1 below.

In a parallel literature on adjustment costs in firm investment, Khan and Thomas (2008) show that even though dynamics at the firm level are highly nonlinear, endogenous prices can create aggregate dynamics which are approximately linear. Our habit equivalence result below is connected to this finding. It establishes that a high ratio of idiosyncratic risk to aggregate risk can also create aggregate dynamics which are approximately linear in our exogenous-price setting. In the Khan and Thomas model, the nonlinearities generated by simultaneous adjustment of many firms are infrequent because relative prices adjust such that the benefits of adjustment are limited. In our model, simultaneous adjustment by many agents is infrequent because idiosyncratic risk keeps the cross-sectional distribution near its steady-state shape. The fact that price effects push in the same direction as idiosyncratic risk suggests that even when the price of the commitment good is endogenized - an important issue we leave for future research - the results on approximate linearity are not likely to be overturned.

Preferences. When $\kappa \rightarrow \infty$, our model converges to a neoclassical model without adjustment costs, and when $\kappa=0$ we obtain a model with only commitment consumption, as in Grossman and Laroque (1990). Because utility is time-separable, $\gamma$ measures the elasticity of intertemporal substitution as well as relative risk aversion for an individual who is free to adjust both $x$ and $a$. We use this functional form to make the evolution of commitments tractable. However, we believe that the intuitions underlying our main results apply more generally to other specifications as well.

\subsection{Household Behavior}

Optimal choice of commitment and adjustable consumption. The following proposition characterizes the choice of commitment consumption using an $(\mathrm{S}, \mathrm{s})$ band. This result has been previously 
established for a class of models that nests our model as a special case (Flavin and Nakagawa 2008). We state the proposition here as a reference.

Proposition 1 [Household behavior] There exist $s<s^{*}<S$ such that $x_{t}^{i}$ is not adjusted as long as $x_{t}^{i} / w_{t}^{i} \in(s, S)$, but adjusted otherwise; and when it is adjusted, the household sets $x_{t}^{i}=s^{*} w_{t}^{i}$.

The behavior of adjustable consumption $a_{t}^{i}$ can be characterized directly from the Euler equation. The appendix shows that $\log a_{t}$ is a random walk with drift that satisfies

$$
d \log a_{t}^{i}=\mu_{a} \cdot d t+\frac{\pi}{\gamma \sigma} \cdot d z_{t}+\frac{\pi_{E}}{\gamma \sigma_{E}} \cdot d z_{t}^{i}
$$

Here, $\mu_{a}$ is the constant mean growth rate, while the second and third terms measure how $a_{t}$ responds to aggregate shocks $d z_{t}$ and idiosyncratic shocks $d z_{t}^{i}$. Motivated by (5), we define $\sigma_{A}=$ $\pi /(\gamma \sigma)$ and $\sigma_{I}=\pi_{E} /\left(\gamma \sigma_{E}\right)$, which measure the standard deviation of adjustable consumption due to aggregate respectively idiosyncratic risk. Let $\sigma_{T}^{2}=\sigma_{A}^{2}+\sigma_{I}^{2}$ measure total consumption risk.

Characterizing consumption dynamics. Proposition 1 and equation (5) do not constitute a full characterization of consumption dynamics because the $(\mathrm{S}, \mathrm{s})$ rule involves the commitments-towealth ratio, and, by equation (4), the evolution of wealth depends on portfolio decisions. We obtain a full characterization of optimal consumption dynamics by specifying the household's choice of $x_{t}^{i}$ as a function of $a_{t}^{i}$ instead of $w_{t}^{i}$. Define $y_{t}^{i}=\log \left(x_{t}^{i} / a_{t}^{i}\right)$. It then follows from Proposition 1 that there exist numbers $L<M<U$ such that for $y_{t}^{i} \in(L, U)$, the household does not adjust $x_{t}^{i}$ from its prior level; but as soon as $y_{t}^{i}$ reaches $L$ or $U$, the household resets $x_{t}^{i}$ so that $y_{t}^{i}=M{ }^{8}$ This rule characterizes the choice of $x_{t}^{i}$ with an inaction region over $y_{t}^{i}$. Importantly, because $y_{t}^{i}$ depends on the endogenous variable $a_{t}^{i}$, this rule is a description of optimal behavior. However, in combination with (5), which characterizes the evolution of $a_{t}^{i}$, this description yields a complete characterization of consumption dynamics. In particular, given initial values for wealth $w_{0}^{i}$ and commitment $x_{0}^{i}$, the household chooses the initial level of adjustable consumption $a_{0}^{i}$ based on the long-run budget constraint, and the evolution of $a_{t}^{i}$ and $x_{t}^{i}$ are then completely pinned down.

A key implication of this characterization is that household consumption $c_{t}^{i}=a_{t}^{i}+x_{t}^{i}$ jumps on adjustment dates. Chetty and Szeidl (2007) document evidence consistent with this prediction and with the $(\mathrm{S}, \mathrm{s})$ policy predicted by Proposition 1. Using data from the Panel Study of Income

\footnotetext{
${ }^{8}$ The existence of an inaction region representation with $x / a$ follows from the fact that the consumption function $a_{t}^{i}=a\left(w_{t}^{i}, x_{t}^{i}\right)$ is strictly increasing in $w_{t}^{i}$ and homogenous of degree one. As a result it can be used to map the (S,s) band over wealth into a band over adjustable consumption: for example $L=1 / a(1 / s, 1)$.
} 
Dynamics, they show that following "small" unemployment shocks that generate a wage income loss of less than 33 percent, most households cut food consumption significantly, while 31 percent of them move out of their house and adjust housing consumption discretely. In response to larger shocks (wage loss greater than 33 percent), households are more likely to adjust on both margins, and in particular 40 percent of them move and change housing consumption discontinuously.

Interpreting $a_{t}$ as permanent income. As shown by equation (5), $\log a_{t}$ follows a random walk: it adjusts immediately and fully to both aggregate and idiosyncratic shocks. In fact, for an agent facing no adjustment $\operatorname{costs}\left(\lambda_{1}=\lambda_{2}=0\right)$ equation (5) would also characterize the dynamics of total consumption, and hence $a_{t}$ is proportional to what consumption (equivalently, permanent income) would be in the absence of adjustment costs. Thus $a_{t}^{i}$ is usefully thought of as a measure of the permanent income of agent $i$. Given the equivalent characterization of the optimal policy described above, we often take the perspective that $a_{t}^{i}$, defined by (5), measures fluctuations in permanent income, and that $x_{t}^{i}$ evolves in response to these fluctuations.

Initial conditions. We assume that at $t=0$ initial wealth and commitment consumption levels are such that households are all inside their inaction region, that $a_{0}^{i}=A_{0}$ is the same for all households, and that the distribution of $y_{0}^{i}$ inside the $(L, U)$ region is given by $F_{0}(y)$.

\subsection{Existence of a Representative Consumer}

We now show that aggregate dynamics in the adjustment cost model coincide with those of a single-agent economy in which aggregate commitments act as a habit-like reference point for the representative consumer. Let $X_{t}=\int_{i} x_{t}^{i} d i, A_{t}=\int_{i} a_{t}^{i} d i$, and $C_{t}=X_{t}+A_{t}$ denote aggregate commitment, adjustable, and total consumption at time $t$.

Proposition 2 Assume that $\delta=\rho-\frac{\pi_{I}^{2}}{2 \sigma_{I}^{2}}\left(1+\frac{1}{\gamma}\right)>0$. Then the aggregate dynamics of consumption are the optimal policy of a representative consumer with external habit formation utility

$$
E \int_{0}^{\infty} e^{-\delta t} \frac{\left(C_{t}-X_{t}\right)^{1-\gamma}}{1-\gamma} d t
$$

where $X_{t}$ follow the dynamics of aggregate commitments.

The intuition for the existence of a representative consumer is that - as in Grossman and Shiller (1982) - idiosyncratic shocks cancel in the aggregation. The presence of idiosyncratic risk also increases mean consumption growth, and to compensate for this, the representative consumer must 
be more patient than the individual households. An implication of Proposition 2 is that the functional form for the utility of the representative consumer is identical to the commonly used "additive habit" specification (e.g., Constantinides, 1990, Campbell and Cochrane, 1999). In this framework, the only observational difference in the aggregate between the commitment model and habit formation models comes from the dynamics of $X_{t}$.

\section{Dynamics of Aggregate Commitments}

We now turn to characterize the evolution of the aggregate commitments $X_{t}$. We set the stage in Section 3.1 by adapting existing results about the cross-sectional distribution to our setting. The new contribution is in the remainder of the section. In Section 3.2 we present our key idea: we represent $X_{t}$ as a moving-average of past shocks, in which the weights are state-dependent impulse responses determined by the cross-sectional distribution at the time of the shock. In Section 3.3 we show that habit models admit an analogous representation in which the weights are state-independent. Finally, in Section 3.4 we identify conditions under which the weights of the commitments model are approximately state-independent, establishing approximate linearity and an equivalence with habit formation.

\subsection{The Cross-Sectional Distribution}

We begin with preliminary results which build on the literature on firm dynamics. Because they have identical preferences, the numbers $\{L, M, U\}$ are the same for all households in the economy. However households face different idiosyncratic shocks and as a result are in general in different locations in the $(L, U)$ region. Characterizing the dynamics of $X_{t}$ thus requires keeping track of the distribution of households. The main object we use for this purpose is the adjustableconsumption-weighted cross-sectional distribution of $y$, defined as $F(y, t)=\left(1 / A_{t}\right) \int_{\left\{i: y_{i}(t)<y\right\}} a_{t}^{i} d i$. This quantity equals the share of total adjustable consumption at date $t$ which is consumed by households $i$ whose $y_{t}^{i}$ is below $y$ in the inaction region $(L, U)$. Given our discussion in Section 2.3 that $a_{t}$ reflects lifetime resources, $F(y, t)$ can be intuitively thought of as measuring how permanent income is distributed inside the inaction region. Note that because at $t=0$ we have $a_{0}^{i}=A_{0}$ for all households, $F(y, 0)=F_{0}(y)$. Let $\mu_{A}$ denote the instantaneous drift of $A_{t}$ and $f(y, t)$ denote the density of $F(y, t)$, the existence and dynamics of which are characterized by the following result. 
Proposition $3 f(y, t)$ exists for all $t>0$ and satisfies the stochastic partial differential equation for $t>0$ and $y \in(L, U)$

$$
d f(y, t)=\left[\left(\mu_{A}+\frac{\sigma_{I}^{2}}{2}\right) \frac{\partial f(y, t)}{\partial y}+\frac{\sigma_{T}^{2}}{2} \frac{\partial^{2} f(y, t)}{\partial y^{2}}\right] d t+\sigma_{A} \frac{\partial f(y, t)}{\partial y} d z
$$

together with the following boundary conditions:

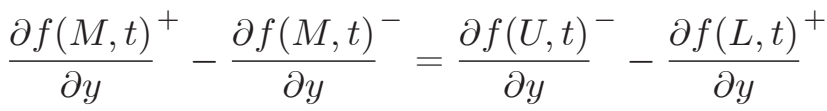

$$
\begin{aligned}
& f(U, t)=f(L, t)=0 \text { and } f(M, t)^{+}=f(M, t)^{-} .
\end{aligned}
$$

Aggregate commitments follow the dynamics

$$
d X_{t}=A_{t} \frac{\sigma_{T}^{2}}{2} \cdot\left(f_{y}(L, t)\left(e^{M}-e^{L}\right)+f_{y}(U, t)\left(e^{U}-e^{M}\right)\right) d t
$$

This result is based on Propositions 1 and 2 in Caballero (1993) combined with Girsanov's theorem to account for a change in drift. Equation (8) shows that the evolution of commitments is "smooth" in the aggregate in the sense that it is a bounded variation process (has no $d z$ term). This follows because the cross-sectional densities go to zero near the boundary of the (S,s) band. As a result the total mass of agents who adjust in response to an aggregate shock of size $d z$ is small: it is proportional to the area under the density at the boundaries, which is of order $(d z)^{2}=d t$.

To understand the intuition for equation (7), first consider the case with no aggregate risk $(d z=0)$. Then the final term on the right hand side vanishes, and the resulting partial differential equation has a unique time-invariant solution $f^{*}$. This density $f^{*}$ can be thought of as the "unperturbed" steady state of the economy. In the presence of aggregate shocks, the actual crosssectional density $f$ is constantly perturbed relative to $f^{*}$, as represented by the $d z$ term in (7); but in the long term the system returns to $f^{*}$ in expectation.

Figure 1 illustrates these results. The top panels plot the steady-state distribution $f^{*}$ in two environments: one with high aggregate and low idiosyncratic risk and the other with low aggregate and high idiosyncratic risk. The bottom panels show the actual cross-sectional distribution sampled twenty times from simulating the two environments. The actual distributions are more similar to the steady state distribution when idiosyncratic risk is high relative to aggregate risk. This observation - which follows because idiosyncratic risk forces the distribution to converge towards $f^{*}$, while aggregate risk pushes it away from $f^{*}$ - plays a key role in our approximation result below. 
(a) High aggregate, low idiosyncratic risk (b) Low aggregate, high idiosyncratic risk
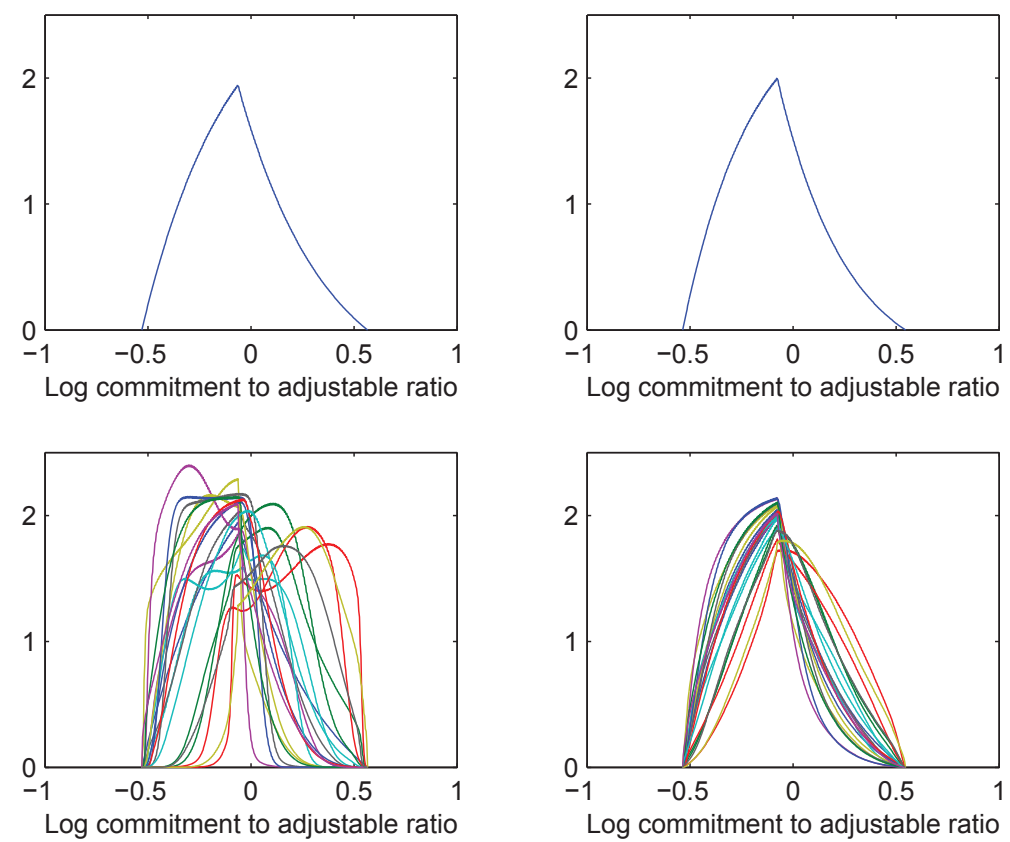

Figure 1: Cross-sectional densities of the log commitment to adjustable consumption ratio. Top panel shows the long run steady state $f^{*}$, bottom panel shows twenty realizations. Environment (a) has high aggregate risk $\left(\sigma_{A}=.1\right)$ and low idiosyncratic risk $\left(\sigma_{I}=.05\right)$, environment (b) has low aggregate risk $\left(\sigma_{A}=.05\right)$ and high idiosyncratic risk $\left(\sigma_{I}=.1\right)$.

\subsection{State-Dependent Impulse Responses and a Moving-Average Representation}

To connect the dynamics of $X_{t}$ to exogenous habit models, we develop a moving average (MA) representation for $X_{t}$. This representation summarizes the dynamic response of $X_{t}$ to past aggregate shocks. Because our interest is in fluctuations, we focus on the de-trended processes $\bar{A}_{t}=e^{-\mu_{A} t} A_{t}$, which is a martingale, and $\bar{X}_{t}=e^{-\mu_{A} t} X_{t}$. It is useful to think of $\bar{A}_{t}$ as summarizing aggregate shocks up to date $t$.

The next definition introduces the impulse response of commitments to an aggregate shock at $t=0$. Specifically, we consider a small change in $A_{0}$ relative to an initial value $A_{0}^{*}$, holding fixed the initial distribution of commitment consumption. Given the initial value $A_{0}^{*}$, the commitment consumption of agent $i$ is $x_{0}^{i *}=a_{0}^{i} \exp y_{0}^{i}=A_{0}^{*} \exp y_{0}^{i}$. Hence -given that the initial distribution of $y_{0}^{i}$ is $F_{0}$ - the initial cross-sectional distribution of commitment consumption is $F^{x}\left(x_{0} \mid A_{0}^{*}\right)=$ $F_{0}\left[\log x_{0}-\log A_{0}^{*}\right] . \quad$ We let $\bar{X}_{t}\left(A_{0}, F^{x}\left(x_{0} \mid A_{0}^{*}\right)\right)$ denote normalized aggregate commitments at date $t$ when $a_{0}^{i}=A_{0}$ may differ from $A_{0}^{*}$, but the initial distribution of commitments is fixed at $F^{x}\left(x_{0} \mid A_{0}^{*}\right)$. 
(a) High aggregate, low idiosyncratic risk

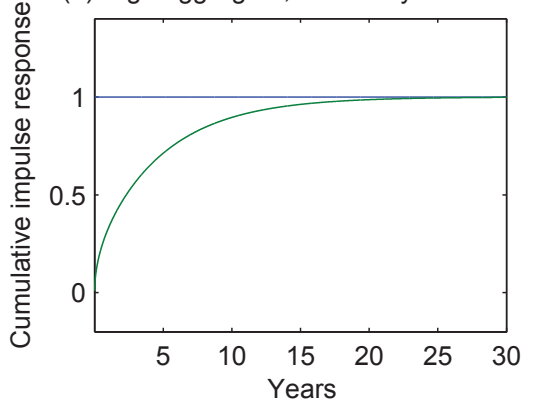

(c) Low aggregate, low idiosyncratic risk

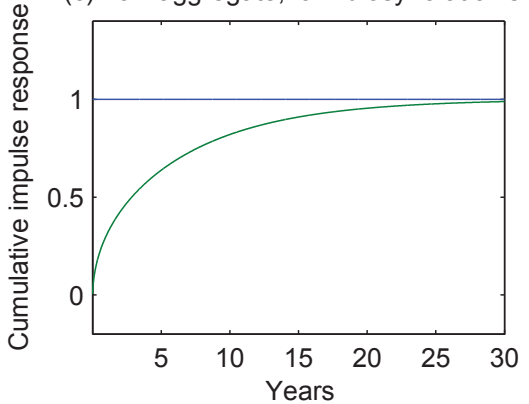

(b) High aggregate, high idiosyncratic risk

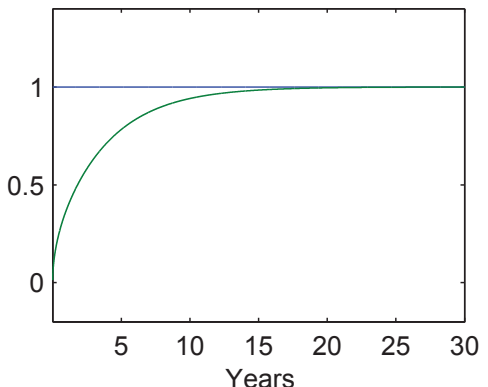

(d) Low aggregate, high idiosyncratic risk

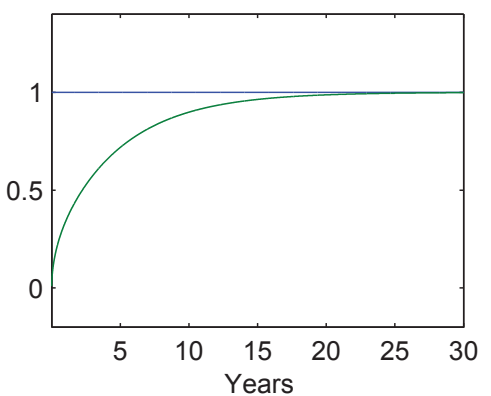

Figure 2: Normalized cumulative impulse response function of aggregate commitment consumption in four environments with high $(\sigma=.1)$ and low $(\sigma=.05)$ aggregate and idiosyncratic risk.

Definition 1 The impulse response function of the commitments model in state $F$ is the function

$$
\xi(t \mid F)=\left.\frac{\partial E_{0}\left[\bar{X}_{t}\left(A_{0}, F^{x}\left(. \mid A_{0}^{*}\right)\right) \mid\right]}{\partial A_{0}}\right|_{F_{0}=F, A_{0}=A_{0}^{*}} .
$$

This is just the derivative of $E_{0} \bar{X}_{t}$ with respect to a uniform change in $a_{0}$ for all households, holding fixed initial commitments. The Appendix shows that $\xi(t \mid F)$ is well-defined and independent of $A_{0}^{*}$. Because we usually work with cross-sectional distributions that have a density, we often write $\xi(t \mid f)$ where $f$ is the density of $F$, or $\xi(t \mid f(s))$ when $f(s)$ is the adjustable-consumptionweighted cross-sectional density at date $s$. It is intuitive that impulse responses should depend on the initial distribution: when many households are on the verge of downsizing, a negative aggregate shock will reduce commitments at a faster rate. Figure 2 plots impulse-responses in our model in four environments (assuming $f=f^{*}$ ). As $t \rightarrow \infty$, these impulse responses gradually converge to a limit (normalized to one in the figures), which corresponds to full adjustment to the initial shock. Higher risk leads to more rapid convergence, as commitments are updated more quickly.

We use $\xi(t \mid f)$ to make explicit the dependence of $X_{t}$ on past aggregate shocks. 
Proposition 4 De-trended aggregate commitments admit the moving average representation

$$
\bar{X}_{t}=\int_{0}^{t} \xi(t-s, f(s)) d \bar{A}_{s}+E_{0} \bar{X}_{t}
$$

As we show below, this MA representation is the key diagnostic in analyzing the dynamics of $X_{t}$. The result is intuitive: the current level of $\bar{X}_{t}$ equals its ex ante expectation plus the sum of the effects of aggregate shocks between date 0 and date $t$, accounting for partial adjustment to shocks using the impulse response function. We interpret (9) as a "state-dependent MA representation" for commitments, where the coefficients $\xi(t-s, f(s))$ depend on the state of the economy at date $s$ through $f(y, s)$.

\subsection{Habit Models and a State-Independent MA Representation}

A leading special case of the moving-average representation in (9) is where the weights $\xi$ are stateindependent, i.e., do not depend on history. We now show that this special case coincides with reduced-form habit models in which $X_{t}$ is specified as an average of past consumption with weights that only depend on the time lag. Intuitively, if habit is a linear function of past consumption, it should be expressible as a linear function of shocks to past consumption as well.

Habit model. Consider a representative agent economy in which external habit preferences are given by (6), and the habit stock is exogenously determined as

$$
X_{t}^{h}=o^{h}(t) X_{0}^{h}+\int_{0}^{t} \zeta^{h}(t-s) C_{s}^{h} d s
$$

with weights $\zeta^{h}$ and $o^{h}$ which are exogenous locally integrable functions asymptoting to zero. Throughout, we follow the convention that the superscript $h$ refers to the representative agent habit model. We assume that the habit consumer has access to the same stock and bond investment opportunities given in equations (3) and (2). Our habit model is therefore a variant of Constantinides (1990). Since the shock processes are identical, we can think of the habit and commitment models as being defined on the same probability space. It is a direct consequence of the Euler equation that in the optimum, the "surplus" consumption $C_{t}^{h}-X_{t}^{h}$ for the habit agent follows the same path as $A_{t}$ in the commitments model. Thus, $A_{t}$ keeps track of aggregate shocks to marginal utility in both economies.

Moving average representation. Lemma 5 in the appendix shows that we can rewrite (10) into a representation in which $X_{t}^{h}$ is a weighted average of past values of $A_{s}$, rather than past values of 
$C_{s}$. This follows essentially because $C, X$ and $A$ are linked by an accounting identity, and hence any linear representation of $X$ in terms of $C$ can also be written as a linear representation in terms of $A$. From that representation, integration by parts yields

$$
\bar{X}_{t}^{h}=\int_{0}^{t} \xi^{h}(t-s) \cdot d \bar{A}_{s}+E_{0} \bar{X}_{t}^{h}
$$

where $\xi^{h}(u)$ is absolutely continuous with respect to the Lebesgue measure. Equation (11) is an MA representation for the detrended habit stock. The fact that the weights in this MA representation are state-independent is a consequence of starting from a habit model in which the consumption weights are state-independent.

\subsection{Equivalence Result: A Fixed-Weight Representation in the Commitments Model}

The results above imply that the central difference between the fixed-weight habit and the commitment models comes from the state-dependent nature of impulse-responses in the latter case. We now show that when the ratio of idiosyncratic to aggregate risk is high, aggregate commitments evolve approximately according to a fixed weight specification.

We begin by introducing a fixed-weight habit model that generates dynamics which match the evolution of commitments on average.

Definition 2 A fixed-weight habit model $X_{t}^{h}$ matches the steady state impulse response of commitments if $\xi^{h}(t)=\xi\left(t, f^{*}\right)$ for all $t$.

In words, we focus on the habit model that has the same impulse responses as the commitment model in its "unperturbed" steady state $f^{*}$. This definition pins down all MA coefficients in (11). We denote the impulse-response weights by $\xi^{*}(u)=\xi\left(u, f^{*}\right)$ and the habit model by $X_{t}^{h *}$.

Main result. Our equivalence result holds when the ratio of idiosyncratic $\left(\sigma_{I}\right)$ to aggregate $\left(\sigma_{A}\right)$ consumption risk is large. Since both of these parameters are endogenous, we study sequences of exogenous parameters such that the implied ratio $\sigma_{I} / \sigma_{A}$ goes to infinity. We explain why $\sigma_{I} / \sigma_{A}$ drives the result in the discussion below. Consider a sequence of models $\Theta^{n}$ such that, as $n \rightarrow \infty$, the following properties hold: 1) $\sigma_{I}^{n} / \sigma_{A}^{n} \rightarrow \infty$; 2) $\gamma, \kappa$ and $\bar{\lambda}_{i}$ remain fixed; 3) $r^{n}$ stays bounded away from zero; 4) $\mu_{A}^{n}$ remains bounded; and 5) $r^{n} / \rho^{n}$ is bounded away from zero and infinity. An example of such a sequence is when $\pi^{n}=1 / n$, while all other exogenous parameters stay constant. In this sequence, $\sigma_{A}^{n} \rightarrow 0$. 

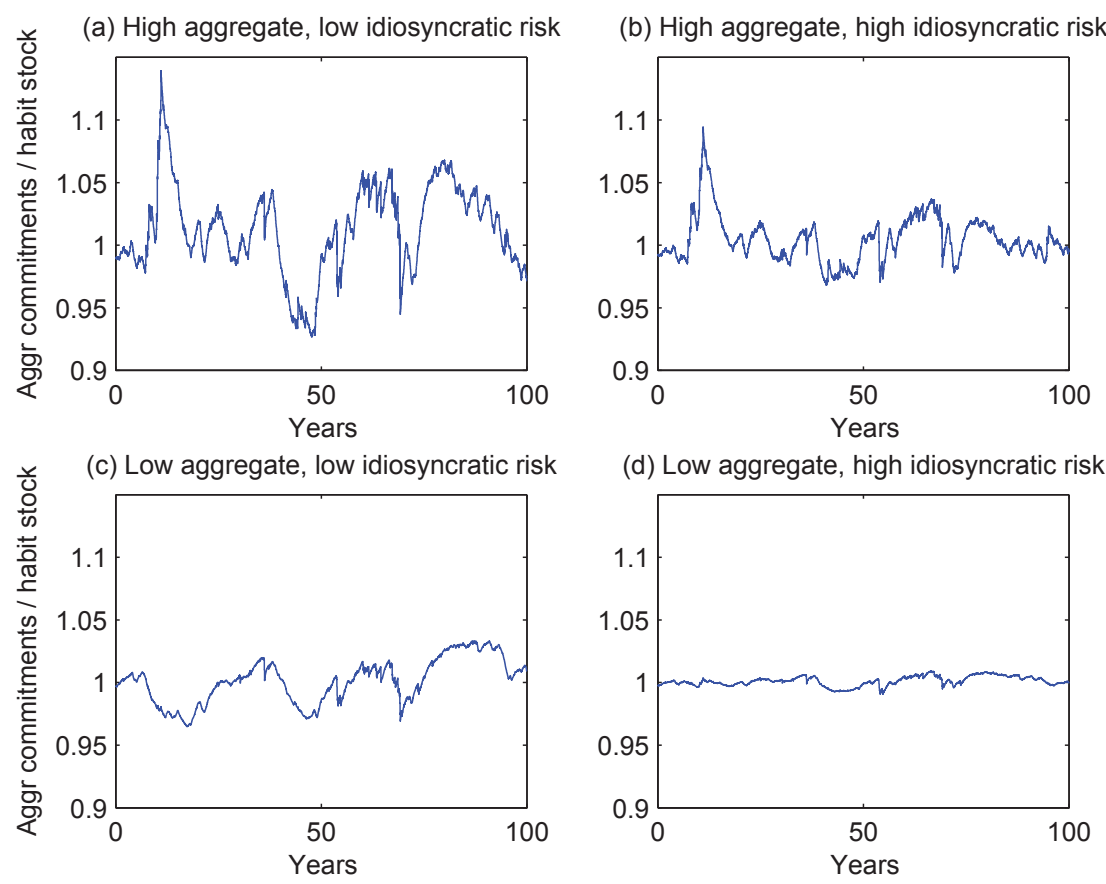

(d) Low aggregate, high idiosyncratic risk

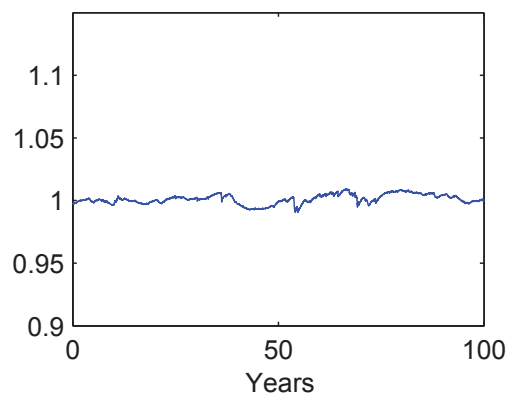

Figure 3: Ratio of aggregate commitments and habit in four environments.

Theorem 1 For any sequence of models $\Theta_{n}$ specified above and any $p \geq 1$,

$$
\lim \sup _{t}\left\|\frac{X_{t}-X_{t}^{h *}}{A_{t}}\right\|_{p}=o\left(\frac{\sigma_{A}}{\sigma_{I}}\right)
$$

The left hand side of the expression measures the distance between aggregate commitments $X_{t}$ and habit in the matching fixed-weight model $X_{t}^{h *}$, rescaled by a measure of the aggregate economy $A_{t}$. Since these quantities are stochastic, we use the $L_{p}$ norm to measure distance, defined as $\|Y\|_{p}=\left[\mathrm{E} Y^{p}\right]^{1 / p}$ for any random variable $Y$. The small order $o($.$) on the right hand$ side shows the accuracy of the approximation: the distance between the two models becomes an arbitrarily small share of $\sigma_{A} / \sigma_{I}$ when this ratio goes to zero. The interpretation is that fixedweight habit provides a highly accurate, "better than first-order" approximation. For example, along a sequence where $\sigma_{A} \rightarrow 0$, the difference between commitments and the fixed-weight model goes to zero even relative to $\sigma_{A}$ : when the size of aggregate shocks shrinks, the approximation error becomes small compared to these shocks. Similarly, when the magnitude of idiosyncratic risk grows, the distance between the two models goes to zero at a faster rate than the growth in $\sigma_{I}$.

Simulations presented in Figure 3 illustrate the theorem. The figure uses a calibration to plot the evolution of $X_{t}^{h *} / X_{t}$ in four environments, in which $\sigma_{I}$ and $\sigma_{A}$ equal either $5 \%$ or $10 \%$. The 
figure shows that the ratio is close to one in most periods, particularly when idiosyncratic risk is high (right panels) and when aggregate risk is low (bottom panels).

The intuition underlying Theorem 1 is that when most of the uncertainty comes from idiosyncratic risk, the cross-sectional distribution is usually close to its steady state. Hence aggregate shocks generate the same pattern of adjustment in most periods, resulting in impulse response weights that are almost constant over time. The proof of the theorem involves several technical steps, but the basic logic is intuitive. The key is to analyze both models using their MA representations. Differencing (9) and (11) yields

$$
\begin{aligned}
\bar{X}_{t}-\bar{X}_{t}^{h *}-E_{0}\left[\bar{X}_{t}-\bar{X}_{t}^{h *}\right] & =\int_{0}^{t}\left[\xi^{*}(t-s)-\xi(t-s, f(s))\right] \cdot d \bar{A}_{s} \\
& =\int_{0}^{t}\left[\xi^{*}(t-s)-\xi(t-s, f(s))\right] \cdot \sigma_{A} \cdot \bar{A}_{s} d z_{s}
\end{aligned}
$$

where we use $d \bar{A}_{s}=\bar{A}_{s} \sigma_{A} d z_{s}$. Focusing on the final integral, consider a sequence of models $\Theta_{n}$ along which the level of aggregate risk $\sigma_{A} \rightarrow 0$. Since the integrand involves $\sigma_{A}$, its value goes to zero as $\sigma_{A} \rightarrow 0$ : as aggregate shocks become small, both models will stay close to their unconditional expectation. But the equation also reveals an additional effect. As $\sigma_{A} / \sigma_{I}$ becomes small, much of the shock each household experiences is idiosyncratic. This pushes the cross-sectional distribution $f$ close to its unperturbed steady state $f^{*}$, because the force pushing for convergence, determined by $\sigma_{I}$, becomes stronger relative to the force of divergence, determined by $\sigma_{A} \cdot{ }^{9}$ As a result, $f$ and $f^{*}$ are usually close. This in turn implies that $\xi^{*}(t-s)-\xi(t-s, f(s))$ is typically small: when the system is close to the steady state, its impulse response is also close to the steady state impulse response. Thus $\bar{X}-\bar{X}^{h}$ is on average small even relative to $\sigma_{A}$.

The mechanism described here is illustrated in the bottom panel of Figure 1. As noted above, there is much more "variance" in the evolution of the cross-sectional distribution in the left panel (low $\sigma_{I} / \sigma_{A}$ ), because the forces of divergence are stronger. This creates fluctuations in the impulseresponse across periods, producing behavior that diverges from a fixed-weight habit model. In contrast, the cross-sectional density varies much less in the right panel. As a result, the impulseresponses are approximately constant, creating approximately linear aggregate dynamics.

The case where $\sigma_{I} / \sigma_{A}$ is large is the most empirically relevant scenario, since idiosyncratic consumption risk is generally much larger than economy-wide risk (e.g., Deaton, 1991, Carroll, Hall, and Zeldes 1992). This suggests commitments can potentially account for behavior typically

\footnotetext{
${ }^{9}$ This mechanism is labeled the "attractor effect" by Caballero (1993).
} 
attributed to habit formation.

\section{Comparing Consumption Commitments and Habit Formation}

In addition to replicating the patterns previously attributed to habit formation models in a commonlystudied environment, the commitments model also yields new predictions in other settings. In this section, we illustrate these predictions using three applications. We discuss how existing evidence and future empirical work can distinguish between the commitment and habit models and derive welfare implications which show why distinguishing the two models is important.

\subsection{Consumption Dynamics}

Two well-documented features of consumption behavior-both in the aggregate and at the micro level - are excess sensitivity and excess smoothness to shocks (see Japelli and Pistaferri (2010) for a review). One major reason for using habit preferences in applied macroeconomic models is that they generate such delayed consumption responses (Fuhrer 2000). In this subsection, we show that the commitments model not only produces these patterns but also matches additional microeconometric evidence on how excess sensitivity depends on the size of the shock and varies across types of consumption.

Fix a date $t_{0}$ and history up to $t_{0}$. For any $t_{1}>t_{0}$ consider the following regression specification for consumption growth:

$$
\log \left(C_{t_{1}}\right)-\log \left(C_{t_{0}}\right)=\alpha_{1}+\beta_{1} \cdot\left[\log A_{t_{1}}-\log A_{t_{0}}\right]+\varepsilon
$$

This regression builds on the interpretation developed in Section 2.3 that adjustable consumptionbecause it immediately and fully responds to shocks - can be thought of as a measure of permanent income for an individual or a group of households. Thus the regression evaluates the extent to which consumption responds to contemporaneous shocks affecting lifetime income. To make explicit its dependence on $t_{1}$, we denote the regression coefficient by $\beta_{1}\left(t_{1}\right)$. The neoclassical permanent income model predicts $\beta_{1}\left(t_{1}\right)=1$ for all $t_{1}>t_{0}$. Following Flavin (1981), we say that consumption

is excessively smooth if $\beta_{1}\left(t_{1}\right)<1$ for some $t_{1}>t_{0}$, i.e., if consumption does not fully respond to contemporaneous shocks. 
Next, let $t_{3}>t_{2}>t_{1}$ and consider the regression

$$
\log C_{t_{3}}-\log C_{t_{2}}=\alpha_{2}+\beta_{2}\left[\log A_{t_{1}}-\log A_{t_{0}}\right]+\varepsilon
$$

This regression evaluates the extent to which consumption adjusts to income shocks with a delay. Using the notation $\left(t_{1}, t_{2}, t_{3}\right)=\bar{t}$, we denote the regression coefficient by $\beta_{2}(\bar{t})$. The neoclassical permanent income model implies $\beta_{2}(\bar{t})=0$ for all $\bar{t}$ because consumption responds fully at the time of the shock. We say that consumption is excessively sensitive if current consumption does respond to past shocks to permanent income, i.e., if there exists $\bar{t}$ such that $\beta_{2}(\bar{t})>0$.

Proposition 5 (Excess smoothness and sensitivity) In the commitments model, consumption is both excessively smooth and excessively sensitive.

Excess smoothness follows because commitments respond slowly to the shock. Therefore initially (for $t_{1}$ close to $\left.t_{0}\right) \beta_{1}\left(t_{1}\right) \approx A_{t_{0}} / C_{t_{0}}<1$ in regression (12). Excess sensitivity is an implication of the fact that eventually, households do adjust their commitments, and hence $\beta_{2}(\bar{t})$ approximates $X_{t_{0}} / C_{t_{0}}>0$ when $t_{2} \rightarrow t_{0}$ and $t_{3} \rightarrow \infty$. The shape of delayed adjustment is illustrated in Figure 2, which plots the normalized steady-state impulse response of commitments. Our model suggests that both the sluggishness and sensitivity of consumption may be consequences of adjustment costs that delay updating.

Large shocks. We now show that excess sensitivity and smoothness vanish for large shocks in the commitments model, but not in the habit model. We first introduce a notion of large shocks. Because our model does not feature jumps, we focus on the (unlikely) events in which $A_{t}$ changes rapidly during a short interval after $t_{0}$. Formally, consider the events in which $\log \bar{A}_{t_{1}}$ reaches either $\log \bar{A}_{t_{0}}+\Delta$ or $\log \bar{A}_{t_{0}}-\Delta$ by date $t_{1}$. These events correspond to a positive (respectively negative) shock, and $\Delta$ measures the size of the shock, i.e., the percentage change in $\bar{A}_{t}$. We denote the former event by $S\left(+, t_{1}, \Delta\right)$, the latter event by $S\left(-, t_{1}, \Delta\right)$, and their union by $S\left(t_{1}, \Delta\right) .{ }^{10}$

We now compare the commitment model with its matching habit specification introduced in Definition 2 during and after these large shocks. Consider estimating the regression (12) conditional on the shock event $S\left(t_{1}, \Delta\right)$. We denote the regression coefficients by $\beta_{1}\left(t_{1}, \Delta\right)$ in the commitments model and $\beta_{1}^{h}\left(t_{1}, \Delta\right)$ in the habit model. Note that because these coefficients are estimated conditional on the low-probability shock events, they need not match the unconditional coefficients $\beta_{1}\left(t_{1}\right)$ and $\beta_{1}^{h}\left(t_{1}\right)$ introduced earlier.

\footnotetext{
${ }^{10}$ The formal way to model these events is to assume that a Brownian bridge drives $\log \bar{A}_{t}$ between $t_{0}$ and $t_{1}$.
} 
Proposition 6 (Excess smoothness for large shocks) The following statements hold:

(i) In the commitments model excess smoothness vanishes for large shocks. Formally, there exists $K>0$ such that for all $t_{1}>t_{0}, \beta_{1}\left(t_{1}, \Delta\right)>1-K / \Delta$.

(ii) In the habit model excess smoothness remains for large shocks. Formally, there exists $K^{\prime}<1$ such that for all $\Delta$ large enough, we can find $t_{1}$ for which $\beta_{1}^{h}\left(t_{1}, \Delta\right)<K^{\prime}$.

Part (i) shows that in the commitments model the correlation between consumption and permanent income increases in extreme events. Because large shocks force people to adjust their commitments, $\beta_{1}\left(t_{1}, \Delta\right)$ approaches 1 as $\Delta \rightarrow \infty$. Part (ii) shows that this result does not extend to the habit model: because in that setting impulse responses do not depend on the size of the shock, $\beta_{1}^{h}\left(t_{1}, \Delta\right)$ remains bounded below 1 even for $\Delta$ large.

We now turn to excess sensitivity. Consider estimating the regression (13) conditional on the shock event $S\left(t_{1}, \Delta\right)$. We denote the regression coefficients by $\beta_{2}(\bar{t}, \Delta)$ in the commitments model, and by $\beta_{2}^{h}(\bar{t}, \Delta)$ in the habit model. To explore the impact of a sudden large shock, we focus on the limit in which, holding fixed $\Delta$ the size of the shock, $t_{1} \rightarrow t_{0}$. We define the $\lim \sup$ and $\lim$ inf of the regression coefficients to be $\bar{\beta}_{2}\left(t_{2}, t_{3}, \Delta\right)=\lim _{t_{1} \rightarrow t_{0}} \sup \beta_{2}(\bar{t}, \Delta)$ and $\underline{\beta}_{2}\left(t_{2}, t_{3}, \Delta\right)=\lim _{t_{1} \rightarrow t_{0}} \inf \beta_{2}(\bar{t}, \Delta)$ in the commitments model, and define $\bar{\beta}_{2}^{h}\left(t_{2}, t_{3}, \Delta\right)$ and $\underline{\beta}_{2}^{h}\left(t_{2}, t_{3}, \Delta\right)$ analogously for the habit model.

We consider a sequence of models $\Theta_{n}$ as defined in Section 3. The following result is stated for the case when $n$ is large enough, that is, when $\sigma_{A} / \sigma_{I}$ is small enough. We focus on this case for the technical reason that it ensures that $X_{t}^{h} / A_{t}^{h}$ remains bounded in $L_{p}$ norm uniformly in $t$.

Proposition 7 (Excess sensitivity for large shocks.) Suppose that $n$ is large enough. Then:

(i) In the commitments model, excess sensitivity vanishes for large shocks. Formally, there exists $K>0$ such that for any $t_{3}>t_{2}$, we have $\bar{\beta}_{2}\left(t_{2}, t_{3}, \Delta\right)<K / \Delta$.

(ii) In the habit model excess sensitivity remains for large shocks as well. Formally, there exists $K^{\prime}>0$ such that for all large enough $\Delta$, we can find $t_{2}$ and $t_{3}$ for which $\underline{\beta}_{2}^{h}\left(t_{2}, t_{3}, \Delta\right)>K^{\prime}$.

Part (i) shows that the commitments model does not generate delayed adjustment for large shocks. As more and more households are pushed over the boundary of their $(\mathrm{S}, \mathrm{s})$ bands, fewer and fewer of them will adjust to the shock with a lag. As a result, $\bar{\beta}_{2}\left(t_{2}, t_{3}, \Delta\right)$ becomes arbitrarily small as $\Delta$ grows. Conversely, part (ii) shows that-because impulse responses are state-independentthe habit model produces delayed responses for large shocks as well. 
The challenging part of the proof is claim (ii). To establish that result, we need to characterize $X_{t_{3}}^{h} / A_{t_{3}}$ as $t_{3} \rightarrow \infty$. Since $X_{t_{3}}^{h}$ is essentially a weighted sum in which the number of terms grows with $t_{3}$, to obtain a characterization we need to make sure that terms corresponding to the distant past, even when normalized by $A_{t_{3}}$, remain bounded. Because $\sigma_{A}$ governs the variance of the normalizing term $A_{t_{3}}^{h}$, while $\sigma_{I}$ affects the rate with which the weights in the weighted sum approach zero, this is ensured when $\sigma_{A} / \sigma_{I}$ is small.

Microeconometric evidence for the "magnitude hypothesis." Summarizing the empirical literature on consumption, Japelli and Pistaferri (2010) write that consumers "tend to smooth consumption and follow the [neoclassical] theory when expected income changes are large, but are less likely to do so when the changes are small and the cost of adjusting consumption is not trivial." Japelli and Pistaferri term this pattern the "magnitude hypothesis." In what follows, we briefly summarize this body of evidence and discuss how it is explained by the commitments model.

Several empirical studies have found that the degree of excess sensitivity in consumption - often measured with the consumption response to anticipated income shocks - depends on the size of the shock. Hsieh (2003) finds that Alaskan households increase consumption in the quarter in which they receive their tax refunds (a small income change), but not in the quarter in which they receive payments from the Alaska Permanent Fund (a large income change). In the same spirit, Browning and Crossley (2001) note that Parker (1999) finds excess consumption sensitivity to the income change associated with US households reaching the Social Security payroll cap (a small income change) while Browning and Collado (2001) find no excess consumption sensitivity of Spanish workers to anticipated bonus salary amounting to two months' wages (a large income change). In support of the idea that the magnitude of the shock may drive these differences, Browning and Crossley (2001) estimate that the welfare cost of ignoring the Spanish bonus system is equivalent to an annual loss of a month's consumption, that of ignoring the Alaska Permanent Fund schedule is equivalent to a week of consumption, and that of the Social Security cap is equivalent to an afternoon's consumption. Similarly, Souleles (1999) finds excess sensitivity to tax refunds and Souleles (2002) to the Reagan tax cuts, but Souleles (2000) finds no excess sensitivity to college expenditures, which are typically larger in magnitude. Finally, Scholnick (2013) shows that the anticipated income increase associated with a household's final mortgage payment has a positive effect on consumption, but the effect is decreasing in the size of the payment.

The commitments model can help explain this body of evidence through Propositions 6 and 7 , which together imply that the delay with which consumption responds to income shocks is smaller 
for large shocks. ${ }^{11}$ This result can explain Hsieh's findings through the logic that consumers respond slowly to information on tax refunds, because those payments are small. But the same consumers respond quickly to news about the payment of the Alaska Permanent fund because those payments are large. In particular, through Proposition 6 the commitments model predicts that consumers should not increase consumption when the actual payment of the Alaska Permanent Fund is made; instead, they should increase consumption earlier, right after the announcement. This prediction is consistent with Hsieh's finding that the growth in expenditures on durables is lower when the Alaska Permanent Fund payment is higher, suggesting that consumers purchase durables before they receive the Fund payment. Similarly, the commitments model predicts slow adjustment to the small income change associated with the relatively small tax refunds and with reaching the social security cap; but like the permanent income model, early adjustment - when the worker is hired, or when a decision is made that the child will attend college - to the wage bonuses and to college expenditures. The habit model does not match these predictions because it produces a state-independent impulse response, as shown in Propositions 6 and $7 .{ }^{12}$

An important caveat is that both the commitment and habit models predict that consumption should be unaffected by the timing of income conditional on the announcement date. Both models simply predict gradual adjustment after the announcement, which results in comovement between income and consumption. Thus neither model can explain the findings of Johnson, Parker and Souleles (2006) that consumption responds to variation in the timing of income tax rebates. Other factors, such as credit constraints (Agarwal, Liu and Souleles, 2007) or salience effects (Bordalo et al. 2012, Koszegi and Szeidl 2013) may help explain this behavior. Despite these caveats, it is clear that important elements of the evidence on excess sensitivity are more consistent with a model of adjustment costs than with habits, suggesting that at least part of the behavior previously attributed to habit formation may in fact be due to consumption commitments.

\subsection{Comparative Dynamics}

In this subsection, we compare the effects of changes in the environment in the commitment and habit models. In the habit model, the weights that determine the speed of adjustment are exogenous

\footnotetext{
${ }^{11}$ Here we use the aggregated commitments model to match micro evidence. The interpretation is that the theoretical aggregate corresponds to the group of households who experience the shock.

${ }^{12}$ In the commitments model, total consumption $C_{t}$ exhibits excess sensitivity and smoothness, while adjustable consumption $A_{t}$ does not. Since most consumption goods have both adjustable and fixed components, the more general empirical prediction is that more adjustable goods exhibit less excess sensitivity and smoothness. This prediction also accords with empirical evidence. For instance, Chetty and Szeidl (2007) find that consumption of housing responds much more sluggishly to unemployment shocks than consumption of food.
} 


\begin{tabular}{|c|c|c|c|c|c|c|}
\hline $\begin{array}{l}\text { Aggregate } \\
\text { risk }\end{array}$ & $\begin{array}{c}\text { Idiosyncratic } \\
\text { risk }\end{array}$ & $\begin{array}{l}\text { Riskfree } \\
\text { rate }\end{array}$ & $\begin{array}{l}\text { Individ cons } \\
\text { growth }\end{array}$ & $\begin{array}{c}\text { How many } \\
\widetilde{p}=0.25\end{array}$ & $\begin{array}{c}\text { years till } X \\
\widetilde{p}=0.5\end{array}$ & $\begin{array}{r}\text { adjusts } \widetilde{p} ? \\
\widetilde{p}=0.75\end{array}$ \\
\hline \multicolumn{7}{|c|}{ Adjustment cost $=1 \times$ annual consumption } \\
\hline $10 \%$ & $10 \%$ & $1 \%$ & $0.87 \%$ & 0.5 & 1.8 & 4.5 \\
\hline $5 \%$ & $10 \%$ & $2.5 \%$ & $0.87 \%$ & 0.6 & 2.2 & 5.6 \\
\hline $10 \%$ & $5 \%$ & $2.5 \%$ & $0.87 \%$ & 0.6 & 2.3 & 5.7 \\
\hline $5 \%$ & $5 \%$ & $4 \%$ & $0.87 \%$ & 0.8 & 3.4 & 8.7 \\
\hline $10 \%$ & $10 \%$ & $4 \%$ & $2.37 \%$ & 0.4 & 1.6 & 4.2 \\
\hline \multicolumn{7}{|c|}{ Adjustment cost $=5 \times$ annual consumption } \\
\hline $10 \%$ & $10 \%$ & $1 \%$ & $0.87 \%$ & 1.1 & 4.2 & 10.3 \\
\hline $5 \%$ & $10 \%$ & $2.5 \%$ & $0.87 \%$ & 1.2 & 4.9 & 12.8 \\
\hline $10 \%$ & $5 \%$ & $2.5 \%$ & $0.87 \%$ & 1.5 & 5.7 & 14 \\
\hline $10 \%$ & $10 \%$ & $4 \%$ & $2.37 \%$ & 0.7 & 3.1 & 8.4 \\
\hline
\end{tabular}

Table 1: Speed of adjustment of consumption commitments

and unaffected by environmental changes. In contrast, in the commitments model household adjustment behavior is endogenous and responds to environmental changes.

To characterize how responses to shocks vary with the environment, let $T(\widetilde{p}, f)=\inf _{t}\{\xi(t \mid f) \geq \widetilde{p} \cdot \bar{x}\}$ denote the time required for commitments or habit to adjust, in expectation, a share $\widetilde{p}$ to a unit shock to permanent income. ${ }^{13}$ This quantity can be interpreted as a measure of excess sensitivity of consumption. By definition, in a fixed-weight habit model, $T(\widetilde{p})$ is pinned down by the exogenous weights and hence remains constant when other parameters are varied.

We begin with some numerical examples to illustrate the comparative dynamics of the commitments model. Table 1 reports $T\left(\widetilde{p} \mid f^{*}\right)$ for the commitments model when $\widetilde{p}=0.25,0.5$ and 0.75 for various parameters. In the top panel, the adjustment cost equals one year's consumption value of the commitment good, or $1 \%$ of its capitalized value with a riskfree rate of $1 \%$. The first row shows that when $\sigma_{A}=\sigma_{I}=10 \%$ and $r_{f}=1 \%$, it takes on average about 1.8 years for $50 \%$ of full adjustment to occur. The next three rows illustrate the effect of reducing $\sigma_{A}$ or $\sigma_{I}$, changing $r_{f}$ so that expected consumption growth remains unchanged in these comparisons. The table shows that reducing either idiosyncratic or aggregate risk results in a slower response to shocks. The intuition is that higher risk forces consumers to update their commitments more frequently, allowing aggregate shocks to get absorbed by commitment consumption more quickly. Comparing the first and last rows in the top panel shows the effect of higher consumption growth generated by a higher safe return. Faster growth also leads to faster adjustment to shocks, as agents update commitments more frequently in a growing economy. The bottom panel of the table shows that

\footnotetext{
${ }^{13}$ Here, $\bar{x}$ denotes the steady state ratio of commitments to adjustables, so that $\xi(t) / \bar{x}$ asymptotes to one.
} 
for a higher adjustment cost (5\% of the capitalized value of the commitment good), adjustment is more sluggish, but the effects of risk and growth remain similar.

To demonstrate that these results are driven by the intuition we describe, we now establish a formal analog of the preceding numerical examples in a special case of the model. Consider a sequence of economies $\bar{\Theta}^{n}$ with $n=1,2, \ldots$ in which $\pi^{n}=\pi_{E}^{n}=1 / n$ and $r=\rho$. This sequence is a special case of the $\Theta_{n}$ sequence introduced earlier in which $\sigma_{I}, \sigma_{A}, \mu_{a}$ and $\mu_{A}$ all go to zero at a rate of $1 / n$. When $n$ grows large, this economy converges to an environment in which households face no risk and have zero consumption growth, which we denote by $\bar{\Theta}^{*}$. Clearly, in that limit economy agents either adjust commitments immediately at $t=0$ or never do so. The habit model that matches the consumption pattern of $\bar{\Theta}^{*}$ (as given by Definition 2) is one in which the habit stock remains unchanged at the initial level of commitment $x_{0}$ forever.

Proposition 8 Fix $\widetilde{p}>0$. In the commitments model, $T^{n}\left(\widetilde{p} \mid x_{0}\right)$ is finite but $\lim _{n \rightarrow \infty} T^{n}\left(\widetilde{p} \mid x_{0}\right)=$ $\infty$. In the habit model, $T^{h, n}\left(\widetilde{p} \mid x_{0}\right)=\infty$ for all $n$.

In the commitments model, adjustment occurs with positive risk and growth ( $n$ finite), but as $n \rightarrow \infty$, it occurs at a vanishingly small rate, so that the expected time to adjustment converges to infinity. In contrast, in the habit model, the presence of risk and growth does not affect adjustment of the habit stock, which remains constant permanently.

At the macroeconomic level, Proposition 8 suggests that recessions may be shorter in rapidly developing economies, in which households change their arrangements frequently because of high trend growth. Conversely, recessions may be longer in economies with substantial social insurance against idiosyncratic risk (such as European welfare states) because people have weaker incentives to change their commitments. Future research testing these predictions would help further distinguish between the commitments and the habit model as drivers of excess consumption sensitivity.

\subsection{Welfare Costs of Shocks}

In our final application, we briefly explore the welfare implications of the commitment and habit models. To begin, note that the commitments model offers a natural welfare measure based on expected utility. In contrast, in the habit model, the appropriate measure is open to debate: in particular, should habit consumption be included in welfare calculations? Following prior work (e.g., Ljungqvist and Uhlig 2000, Ljungqvist and Uhlig 2009), we assume that the welfare of the habit agent is fully determined by surplus consumption, without including the habit stock itself. 


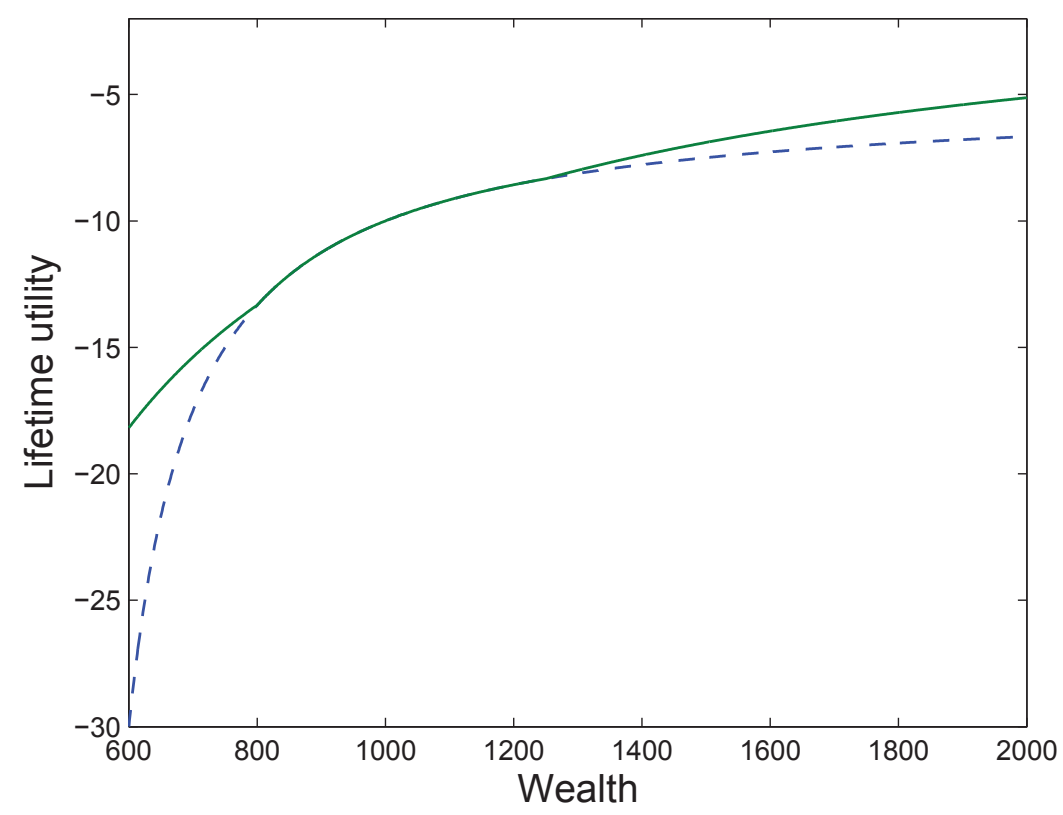

Figure 4: Value (lifetime utility) as a function of wealth of a commitment agent (solid line) and the matching habit agent (dashed line) in an economy with zero consumption risk and no growth. The value function of the habit agent is shifted vertically to account for the utility value of commitments.

This assumption fits with the neoclassical tradition of assuming that the agent's objective is to maximize his own welfare. It would be useful to examine other welfare measures in future work.

We measure the welfare cost of a one-time, unanticipated wealth shock using a certaintyequivalent approach. We ask what certain reduction in wealth the agent would accept to avoid the risk of experiencing the wealth shock in a given instant of time. ${ }^{14}$ Because this measure is denominated in units of wealth, it can be used to make welfare comparisons across models.

To build intuition, we first focus on the economy $\bar{\Theta}^{*}$ defined earlier, in which there is no aggregate or idiosyncratic risk and no consumption growth $\left(\pi=\pi_{E}=0\right.$ and $r=\rho$, implying $\left.\mu_{a}=\sigma_{A}=\sigma_{I}=0\right)$. Consider Figure 4, which plots the value functions of the commitment and habit agents in this environment. As long as it remains optimal for the commitment agent not to move, the two value functions are parallel. ${ }^{15}$ In this range, all changes in wealth are absorbed by adjustable consumption, and hence the welfare implications of the two models are identical. However, for large shocks, the commitment agent adjusts on both consumption margins, while

\footnotetext{
${ }^{14}$ Focusing on unanticipated shocks allows us to rule out precautionary behavioral responses, simplifying computations.

${ }^{15}$ There is a difference in the level of utility because here we assume that the habit agent does not derive utility from commitment consumption. The figure abstracts away from this effect by shifting the value function of the habit agent vertically.
} 
adjustment of the habit stock is not permitted. As a result, large shocks have a higher welfare cost with habits than with commitments.

To establish this intuition in a more general setting, we consider an unanticipated wealth shock at time $t$ that hits with probability $q$ and reduces total wealth by a share $b$. Consider the commitment economy in its unperturbed steady state in which all agents face this shock, and contrast it with the matching habit model where the shock affects the representative agent. We define the proportional risk premium $\Pi(q, b)$ in either model as the dollar amount that agents in that model are collectively willing to give up in excess of the expected value to avoid this risk, normalized by total wealth in the economy.

Proposition 9 Assume that $\lambda_{1}=0$ but $\lambda_{2}>0$. Then:

(i) As $b \rightarrow 1$, the proportional risk premium in the fixed-weight habit economy exceeds that in the corresponding commitment economy: $\Pi_{h}(q, b)>\Pi(q, b)$.

(ii) Consider the sequence of economies $\bar{\Theta}_{n}$. For $b>0$ sufficiently small, in the commitment model the risk premium $\Pi^{n}(q, b)<\Pi^{*}(q, b)$, while in the habit model $\Pi^{h, n}(q, b)=\Pi^{h *}(q, b)$.

Part (i) implies that habit agents are more averse to large shocks than are commitment agents. Commitments adjust immediately to a big shock, mitigating its impact. In contrast, reduced-form habits adjust sluggishly for all shocks, hence agents suffer relatively more from a large shock. ${ }^{16}$

Part (ii) explores comparative statics of the welfare cost as risk and growth vanish. With commitments, risk and growth reduce the risk premium $\Pi(q, b)$ : since agents adjust for other reasons, a shock can be partly absorbed by commitments. Because this possibility is absent in the reduced-form habit model, there the risk premium is unaffected by changes in risk or growth.

A policy lesson from (i) is that a habit model that matches observed dynamics of consumption well may nevertheless yield misleading conclusions about the welfare costs of large shocks. In particular, the optimal size of social insurance programs that insure large, long-term shocks such as disability or job displacement may be smaller than predicted by analyses using habit models such as Ljungqvist and Uhlig (2000). Result (ii) implies that policies which increase social insurance or reduce growth can make aggregate fluctuations more costly by slowing households' response

\footnotetext{
${ }^{16}$ The assumption that $\lambda_{1}=0$ guarantees that when moving, the commitment agents can get rid of all precommitments. Otherwise, even when moving they would still have promised expenditures of $\lambda_{1} X_{t-}$, which behave like sluggish habits. In simulations, we find that unless $\lambda_{1}$ is very high, the conclusion of the proposition is unaffected. Intuitively, moving costs are much smaller than habit expenditures.
} 
commitments habit

\begin{tabular}{llc}
\hline Behavioral Predictions & & \\
\hline 1. household consumption jumps on adjustment dates & yes & no \\
2. consumption excessively sensitive/smooth for small shocks & yes & yes \\
3. excess sensitivity/smoothness vanishes for large shocks & yes & no \\
4. less excess sensitivity/smoothness for adjustable goods & yes & no \\
5. reducing long-term growth can increase excess sensitivity & yes & no \\
6. insuring idiosyncratic risk can increase excess sensitivity & yes & no \\
\hline \hline Welfare Implications & & yes \\
\hline 1. unambiguous welfare measure & yes & yes \\
2. welfare cost of small shocks amplified & yes & no \\
3. welfare cost of large shocks amplified less & yes & no \\
4. reducing trend growth can increase cost of aggregate shocks & yes & no \\
5. insuring idiosyncratic risk can increase cost of aggregate shocks & & \\
\hline \hline
\end{tabular}

Table 2: The main predictions of the commitments and habit models

to changing circumstances. These results illustrate the potential importance of distinguishing between the commitments and habit models.

\section{Conclusion}

A large literature in macroeconomics has used habit formation in preferences as an explanation for key properties of consumption dynamics, such as the excess sensitivity and smoothness of consumption. In this note, we showed that these properties can also be explained by aggregating a model with adjustment costs at the microeconomic level. We also showed that the commitments model yields new predictions in other domains. We conclude with Table 2 which summarizes the key similarities and differences between the models and helps identify directions for future research.

The first four predictions in Table 2, on the dynamics of consumption and its response to shocks, have been studied in prior empirical research. As discussed above, available evidence on the predictions where the models differ aligns more closely with the commitments model. It would be useful to have more evidence on the mechanism underlying these predictions. For example, the commitments model predicts that excess sensitivity should be greater for small shocks (such as lottery winnings) than large shocks, particularly for less adjustable goods like housing or durables. Standard habit models do not predict such heterogeneity.

Predictions 5 and 6 on the impacts of changes in the economic environment offer new ways to distinguish between the two models. One way to test prediction 6 at the microeconomic level would 
be to compare the effect of tax rebates on households who have vs. have not recently experienced a positive income shock, such as a promotion. The commitments model predicts that excess sensitivity of consumption to tax rebates should be lower for those who also had another positive income shock, because they are more likely to adjust for that reason. At the macroeconomic level, prediction 6 suggests that countries with more generous welfare systems, such as those in Northern Europe, should have relatively longer business cycles. ${ }^{17}$

Differentiating between the commitment and habit models is important because the two models generate different welfare implications, listed in the second part of Table 2. If commitments are the root cause of habit-like behavior, then the welfare gains from insuring small or moderate shocks may be larger than the gains from insuring large shocks, especially in economies with low trend growth and idiosyncratic risk. In contrast, if consumers have habit formation preferences, then insuring the largest shocks is most important. More broadly, revisiting existing results on optimal policy in models featuring consumption commitments would be a useful direction for future research.

\section{References}

Ameriks, J., Caplin, A., and Leahy, J. (2003) "Wealth Accumulation and the Propensity to Plan," Quarterly Journal of Economics 118: 1007-1047.

Attanasio, O. P. (2000). "Consumer Durables and Inertial Behavior: Estimation and Aggregation of (S,s) Rules for Automobile Purchases," Review of Economic Studies 67, 667-696.

Bertola, G. and Caballero, R. J. (1990). "Kinked Adjustment Costs and Aggregate Dynamics." in Oliver J. Blanchard and Stanley Fischer, eds., NBER Macroeconomics Annual, Cambridge, MA: MIT Press.

Boldrin, M., Christiano, L. J. and Fisher, J. D. M. (2001). "Habit Persistence, Asset Returns and the Business Cycle," American Economic Review 91: 149-166.

Bordalo, P., Gennaioli, N. and Shleifer, A. (2012) "Salience Theory of Choice Under Risk," Quarterly Journal of Economics,127, 1243-1285.

Caballero, R. J. (1993). "Durable Goods: An Explanation for Their Slow Adjustment," Journal of Political Economy 101, 351-384.

Caballero, R. J. and Engel, E. M. R. A. (1993) "Microeconomic Adjustment Hazards and Aggregate Dynamics." Quarterly Journal of Economics 108, 359-383.

\footnotetext{
${ }^{17}$ Naturally, this prediction is more speculative because of potential general equilibrium effects and other factors that may vary across countries that are missing from our stylized model.
} 
Caballero, R. J. and Engel, E. M. R. A. (1999) "Explaining Investment Dynamics in U.S. Manufacturing: A Generalized (S,s) Approach" Econometrica 67, 783-826.

Campbell, J. Y. and Cochrane, J. H. (1999). "By Force of Habit: A Consumption-based Explanation of Aggregate Stock Market Behavior," Journal of Political Economy 107, 205-251.

Carroll, C. D., Hall, R.E. and Zeldes, S. P. (1992) "The Buffer-Stock Theory of Saving: Some Macroeconomic Evidence." Brookings Papers on Economic Activity 61-156.

Carroll, C. D., Overland, J. and Weil, D. N. (2000). "Saving and Growth with Habit Formation," American Economic Review 90: 341-355.

Chetty, R. and Szeidl, A. (2007). "Consumption Commitments and Risk Preferences," Quarterly Journal of Economics 122(2): 831-877.

Chetty, R. and Szeidl, A. (2014). "The Effect of Housing on Portfolio Choice," Working paper, Harvard University and Central European University.

Christiano, L. J., Eichenbaum, M. and Evans, C. L. (2005). "Nominal Rigidities and the Dynamic Effects of a Shock to Monetary Policy," Journal of Political Economy 113: 1-45.

Constantinides, G. (1990). "Habit Formation: A Resolution of the Equity Premium Puzzle," Journal of Political Economy 98: 519-543.

Deaton, A. (1987). "Life-cycle models of consumption: is the evidence consistent with the theory?" In: Truman, B. (Ed.), Advances in Econometrics, Fifth World Congress, vol. 2. Cambridge University Press, Cambridge.

Deaton, A. (1991). "Saving and Liquidity Constraints," Econometrica 59(5), 1221-1248.

Dybvig, P. H. (1995). "Dusenberry's Racheting of Consumption: Optimal Dynamic Consumption and Investment Given Intolerance for any Decline in Standard of Living," Review of Economic Studies 62: 287-313.

Eberly, J. (1994). "Adustment of Consumers' Durables Stocks: Evidence from Automobile Purchases," Journal of Political Economy 102, 403-436.

Ergin, H. (2003). "Costly Contemplantion," Working paper, Washington University, St Louis.

Flavin, M. (1981) "The Adjustment of Consumption to Changing Expectations about Future Income," Journal of Political Economy, 89, 974-1009.

Flavin, M. and Nakagawa, S. (2008). "A Model of Housing in the Presence of Adjustment Costs: A Structural Interpretation of Habit Persistence," American Economic Review 98: 474-495.

Fratantoni, M. C. (2001). "Homeownership, Committed Expenditure Risk, and the Stockholding Puzzle," Oxford Economic Papers 53: 241-259. 
Fuhrer, J. C. (2000). "Habit Formation in Consumption and Its Implications for MonetaryPolicy Models," American Economic Review 90: 367-390.

Gabaix, X. and Laibson, D. (2001). "The 6D Bias and the Equity Premium Puzzle." NBER Macroeconomics Annual.

Grossman, S. J. and Laroque, G. (1990). "Asset Pricing and Optimal Portfolio Choice in the Presence of Illiquid Durable Consumption Goods," Econometrica 58, 25-51.

Grossman, S. J. and Shiller, R. J. (1982). "Consumption Correlatedness and Risk Measurement in Economies with Non-traded Assets and Heterogeneous Information," Journal of Financial Economics 10: 195-210.

Khan, A. and Thomas, J. (2008). "Idiosyncratic Shocks and the Role of Nonconvexities in Plant and Aggregate Investment Dynamics", Econometrica 76(2), 395-436.

Koszegi, B. and Szeidl, A. (2013). "A Model of Focusing in Economic Choice," Quarterly Journal of Economics

Ljungqvist, L. and Uhlig, H. (2000). "Tax Policy and Aggregate Demand Management under Catching Up with the Joneses," American Economic Review 90: 356-366.

Ljungqvist, L. and Uhlig, H. (2009). "Optimal Endowment Destruction under CampbellCochrane Habit Formation" NBER Working paper 14772.

Lynch, A. (1996). "Decision Frequency and Synchronization across Agents: Implications for Aggregate Consumption and Equity Return." Journal of Finance 51, 1479-1498.

Marshall, D. A. and Parekh, N. G. (1999). "Can Costs of Consumption Adjustment Explain Asset Pricing Puzzles?" Journal of Finance 54, 623-654.

Olney, M. (1999). "Avoiding Default: The Role of Credit in the Consumption Collapse of 1930," Quarterly Journal of Economics 319-335.

Postlewaite, A., Samuelson, L. and Silverman, D. (2008). "Consumption Commitments and Employment Contracts," Review of Economic Studies 75: 559-578.

Reis, R. (2006). "Inattentive Consumers," Journal of Monetary Economics 53: 1761-1800.

Ryder, H. E. and Heal, G. M. (1973). "Optimal Growth with Intertemporally Dependent Preferences," Review of Economic Studies 40: 1-31.

Shore, S. and Sinai, T. (2009). "Commitment, Risk, and Consumption: Do Birds of a Feather Have Bigger Nests?" Review of Economics and Statistics.

Sun, Y. N. (1998) "A theory of hyperfinite processes: the complete removal of individual uncertainty via exact LLN," Journal of Mathematical Economics 29, 419-503. 
Sundaresan, S. M. (1989). "Intertemporally Dependent Preferences and the Volatility of Consumption and Wealth," Review of Financial Studies 3: 73-89.

\section{Appendix A: Proof Map}

We present a series of Lemmas and arguments that build up to the proof of Theorem 1 and to the applications. Additional proofs are contained in the online Supplementary Appendix.

\section{A.1 Preliminaries}

Two convenient probability measures. Let $Q$ be the probabilty measure which weighs the sample paths of $y_{t}$ by their share in aggregate adjustable consumption. Then $F(y, t)=\operatorname{Pr}_{Q}\left[y_{t}^{i}<y \mid A_{[0, t]}\right]$. It follows from the proof (in the Supplementary Appendix) of Proposition 2 that the probability density associated with $Q$ is

$$
\left.\frac{d Q}{d P}\right|_{t}=\frac{a_{t}^{i}}{A_{t}}=\exp \left[\frac{\pi_{I}}{\gamma \sigma_{I}} z_{t}^{i}-\frac{\pi_{I}^{2}}{2 \gamma^{2} \sigma_{I}^{2}} t\right]
$$

which is an exponential martingale. By the Cameron-Martin-Girsanov theorem, under $Q$, the process $d \bar{z}_{t}^{i}=d z_{t}^{i}-\pi_{I} /\left(\gamma \sigma_{I}\right) t$ is a Brownian motion.

For our second probability measure note that - as shown in the proof of Proposition $2-A_{t}$ is an exponential random walk, and hence $\bar{A}_{t}=e^{-\mu_{A} t} A_{t}$ is an exponential martingale. We define a measure $R$ by letting, for any random variable $Z_{t}$ measurable with respect to $\mathcal{F}_{t}, E^{R}\left[Z_{t}\right]=$ $E\left[Z_{t} \bar{A}_{t}\right]$. By the Girsanov theorem, under $R$, the process $d \bar{z}_{t}=d z_{t}-\sigma_{A} t$ is a martingale. The advantage of this measure is that $E_{0} \bar{X}_{t}=E_{0}^{R}\left[\bar{X}_{t} / \bar{A}_{t}\right]$. This makes it easier to compute the mean and the impulse response of $\bar{X}_{t}$, because $\bar{X}_{t} / \bar{A}_{t}$ is a bounded process. We can also write $E_{0} \bar{X}_{t}=E_{0}^{R}\left[\bar{X}_{t} / \bar{A}_{t}\right]=E_{0}^{Q R}\left[x_{t} / a_{t}\right]$ where the superscript $Q R$ means that we first apply the transformation associated with $R$ and then the transformation associated with $Q$. Because the densities associated with these transformations are driven by independent Brownian motions, $Q R$ is also a probability measure. By applying $R$, we move to using the mean dynamics of $\bar{X} / \bar{A}$; and then, by also applying $Q$, we can focus on the mean dynamics of a single agent, albeit under a driving process with different drift.

Limits of models. Theorem 1 takes a sequence of models $\Theta_{n}$. Below we focus on a sequence along which $\sigma_{A} \rightarrow 0$. At the end of the proof we show how to convert this result-using a clock change - to a sequence where $\sigma_{I} \rightarrow \infty$. Along the sequence $\Theta_{n}$, endogenous parameters of the 
model, such as $U$ and $L$, also change. While we do not always indicate it in notation, we always understand those changes to be taking place.

\section{A.2 Auxiliary results about the commitments model}

We begin with a technical lemma that establishes the smoothness of conditional expectations of $y_{t}$. Consider a new process $\widetilde{w}_{t}$, which is a Brownian motion with some drift $\mu_{w}$ and variance $\sigma_{w}$ reborn at some interior point $M_{w}$ when hitting the boundaries of the interval $\left[L_{w}, U_{w}\right]$. With appropriate choice of parameters $\widetilde{w}_{t}$ will have the same distribution as $y_{t}$ under $Q R$. We let

$h\left(y, t, \sigma_{w}, \mu_{w}, L_{w}, M_{w}, U_{w}\right)=E\left[e^{\widetilde{w}_{t}} \mid \widetilde{w}_{0}=y\right]$. Often we just write $h(y, t)$, in which case we assume that the other arguments are given by the optimal policy of the commitments model, so that $h(y, t)=E^{Q R} E\left[e^{y t} \mid y_{0}=y\right]$. Let $L_{1}<L_{2}<M_{1}<M_{2}<U_{1}<U_{2}$.

Lemma $1 h\left(y, t, \sigma_{w}, \mu_{w}, L_{w}, U_{w}, M_{w}\right)$ is infinitely many times differentiable in $\left[L_{w}, U_{w}\right] \times(0, \infty) \times$ $(0, \infty) \times\left[L_{1}, L_{2}\right] \times\left[M_{1}, M_{2}\right] \times\left[U_{1}, U_{2}\right]$.

Thus $h$ and it's various derivatives in $y$ and $t$ are all continuous and therefore locally bounded in $\left(\mu_{w}, \sigma_{w}, L_{w}, M_{w}, U_{w}\right)$. This is useful because when we take $\sigma_{A}$ to zero as $n \rightarrow \infty$, optimal behavior changes, and hence the endogenous parameters $\left(\mu_{y}, \sigma_{y}, L, M, U\right)$ vary. But these parameters will all stay in some bounded open set, and due to positive idiosyncratic risk $\sigma_{y}$ stays bounded away from zero. Thus along this sequence $h(y, t)$ and its derivatives exist and are all bounded.

Our next Lemma expresses $X_{t}$ as a moving average with weights determined by $h$.

Lemma 2 Let $\xi(u, y)=h(u, y)-h_{y}(u, y)$ and $\xi(u, f(s))=\int_{L}^{U} \xi(u, y) f(y, s) d y$. Then

$$
\bar{X}_{t}=\int_{0}^{t} \xi(t-s, f(s)) \sigma \bar{A}_{s} d z_{s}+E_{0}\left[\bar{X}_{t}\right]
$$

Proposition 4 follows from this result. We next show that $\xi(t, y)$ converges exponentially fast.

Lemma 3 There exists $\bar{x}$ such that $\lim _{t \rightarrow \infty} E_{0}\left[\bar{X}_{t}\right]=\lim _{t \rightarrow \infty} \xi(t, y)=\bar{x}$. There exist $K_{1}, K_{2}>0$ independent of $y$ and $\sigma_{A}$ so that $|\xi(t, y)-\bar{x}|<K_{1} e^{-K_{2} t}$ and $\left|E_{0}\left[\bar{X}_{t}\right]-\bar{x}\right|<K_{1} e^{-K_{2} t}$ for all $\left(y, \sigma_{A}\right) \in[L, U] \times\left[0, \bar{\sigma}_{A}\right]$.

The next result will be used in the proof of Theorem 1 to show that for $\sigma_{A}$ small, the impulse responses of the two models are typically close. Let $F^{*}$ denote the invariant distribution of $y$ under $Q$, which is also the long run average cross-sectional distribution of the commitments model. 
Lemma $4 \lim \sup _{t \rightarrow \infty}\left\|\sup _{y}\left|F(y, t)-F^{*}(y)\right|\right\|_{p}$ converges to zero as $\sigma_{A} \rightarrow 0$.

\section{A.3 Auxiliary results about the habit model}

We first show the link between $C$-weighted and $A$-weighted habit models.

Lemma 5 Consider two habit models $X_{t}=\int_{0}^{t} j(t-s) A_{s} d s+k(t) X_{0}$ and $X_{t}=o(t) X_{0}+\int_{0}^{t} \zeta(t-$ $s) C_{s} d s$ where the weight functions $j, k, o$ and $\zeta$ are locally integrable. Then there is a one-to-one correspondence between these representations, and the weights are linked to each other through the Volterra integral equations $\zeta(u)=j(u)-\int_{0}^{u} \zeta(v) j(u-v) d v$ and $o(t)=k(t)-\int_{0}^{t} \zeta(t-s) k(s) d s$ with initial conditions $\zeta(0)=j(0), o(0)=k(0)$. In particular, each $C$-average representation has a unique equivalent A-average representation.

We next construct the best-fit habit model.

Lemma 6 Let $\theta(u)=\xi^{* \prime}(u) \cdot e^{\mu_{A} u}$ and $\theta_{0}(u)=\left(\bar{x}-\xi^{*}(u)\right) \cdot e^{\mu_{A} u}$, then the habit model $X_{t}^{h}=$ $\int_{0}^{t} \theta(t-s) A_{s} d s+\theta_{0}(t) A_{0}$ generates the impulse response $\xi^{*}$.

\section{A.4 Proof of Theorem 1 when aggregate risk vanishes}

We require a technical Lemma bounding the tail of the MA representation in both models.

Lemma 7 Let $g(u, s)$ be progressively measurable with respect to $\mathcal{F}_{s}$ satisfying $|g(u, s)| \leq K_{1} e^{-K_{2} u}$ for all $u, s$, and let $G_{t}=\left(1 / \bar{A}_{t}\right) \int_{0}^{t} g(t-s, s) \bar{A}_{s} d z_{s}$. For any $1 \leq p<\infty$, for $\sigma_{A}$ small enough, there exists $M(p)$ such that $\left\|G_{t}\right\|_{p} \leq M(p)$.

Consider a sequence along which $\sigma_{A} \rightarrow 0$. We can write

$$
\frac{X_{t}-X_{t}^{h}}{\sigma_{A} A_{t}}=\frac{1}{\bar{A}_{t}} \int_{0}^{t}\left[\xi(t-s, f(s))-\xi^{*}(t-s)\right] \bar{A}_{s} d z_{s}+\frac{E_{0} \bar{X}_{t}-\bar{x}}{\bar{A}_{t} \sigma_{A}} .
$$

We now break this expression into three pieces. Fix some $\varepsilon>0$, let $k>0$, and consider

$$
\begin{aligned}
& \left\|\frac{1}{\bar{A}_{t}} \int_{0}^{t-k}\left[\xi(t-s, f(s))-\xi^{*}(t-s)\right] \bar{A}_{s} d z_{s}\right\|_{p} \\
& \quad \leq\left\|\frac{\bar{A}_{t-k}}{\bar{A}_{t}}\right\|_{2 p} \cdot\left\|\frac{1}{\bar{A}_{t-k}} \int_{t-k}^{t}\left[\xi(t-s, f(s))-\xi^{*}(t-s)\right] \bar{A}_{s} d z_{s}\right\|_{2 p} \leq K_{2 p}\left(k, \sigma_{A}\right) \cdot M(2 p) \cdot e^{-K_{2} k}
\end{aligned}
$$

where we used Lemma 7 . We can chose $k$ large enough so that this entire term is less than $\varepsilon / 3$. 
Given this $k$, we next bound the term

$$
\begin{gathered}
\left\|\frac{1}{\bar{A}_{t}} \int_{t-k}^{t}\left[\xi(t-s, f(s))-\xi^{*}(t-s)\right] \bar{A}_{s} d z_{s}\right\| \leq\left\|\frac{\bar{A}_{t-k}}{\bar{A}_{t}}\right\|_{2 p} \cdot\left\|\int_{t-k}^{t}\left[\xi(t-s, f(s))-\xi^{*}(t-s)\right] \frac{\bar{A}_{s}}{\bar{A}_{t-k}} d z_{s}\right\|_{2 p} \\
\leq K_{2 p}\left(k, \sigma_{A}\right) \cdot K_{2 p}(k) \cdot\left[E \int_{t-k}^{t}\left[\xi(t-s, f(s))-\xi^{*}(t-s)\right]^{2 p}\left|\frac{\bar{A}_{s}}{\bar{A}_{t-k}}\right|^{2 p} d s\right]^{1 / 2 p} \\
\leq K_{2 p}\left(k, \sigma_{A}\right) \cdot K_{2 p}(k) \cdot\left[E \int_{t-k}^{t}\left[\xi(t-s, f(s))-\xi^{*}(t-s)\right]^{4 p} d s\right]^{1 / 4 p} \cdot\left[E \int_{t-k}^{t}\left|\frac{\bar{A}_{s}}{\bar{A}_{t-k}}\right|^{4 p} d s\right]^{1 / 4 p} \\
\leq K_{2 p}\left(k, \sigma_{A}\right) \cdot K_{2 p}(k) \cdot K_{4 p}\left(k, \sigma_{A}\right) \cdot\left[E \int_{t-k}^{t}\left[\xi(t-s, f(s))-\xi^{*}(t-s)\right]^{4 p} d s\right]^{1 / 4 p}
\end{gathered}
$$

where we repeatedly used the Cauchy-Schwarz inequality and a martingale moment bound, and where all constants are bounded as $\sigma_{A}$ goes to zero. Next note that

$$
\begin{aligned}
\xi(t-s, f(s))-\xi^{*}(t-s) & =\int_{L}^{U} \xi(t-s, y) \cdot\left[f(t-s, y)-f^{*}(y)\right] d y \\
& =-\int_{L}^{U} \frac{\partial}{\partial y} \xi(t-s, y) \cdot\left[F(t-s, y)-F^{*}(y)\right] d y
\end{aligned}
$$

Here, for any fixed $k$, by Lemma $1, \partial \xi(t-s, y) / \partial y$ is uniformly bounded in $\left(y, \sigma_{A}\right) \in[L, U] \times\left[0, \bar{\sigma}_{A}\right]$. Denoting this bound by $K(k)$, we have

$$
E\left[\xi(t-s, f(s))-\xi^{*}(t-s)\right]^{4 p}<K^{4 p}(k) \cdot E \sup _{y}\left|F(t-s, y)-F^{*}(y)\right|^{4 p} .
$$

Lemma 4 shows that the limsup over $t$ of the last term goes to zero as $\sigma_{A} \rightarrow 0$. Thus given $k$ and $\varepsilon>0$, for all $\sigma_{A}$ small enough to make the entire term bounded above by $\varepsilon / 3$. Finally, consider

$$
\frac{1}{\sigma_{A}} \cdot\left\|\frac{E_{0} \bar{X}_{t}-\bar{x}}{\bar{A}_{t}}\right\|_{p} \leq \frac{1}{\sigma_{A}} \cdot\left\|\frac{1}{\bar{A}_{t}}\right\|_{p} \cdot K_{1} e^{-K_{2} t} \leq \frac{1}{\sigma_{A}} \cdot e^{K_{3}(p) \cdot \sigma_{A}^{2} t} \cdot K_{1} e^{-K_{2} t}
$$

If $\sigma_{A}$ is small enough, then the limsup of this as $t \rightarrow \infty$ is zero.

\section{A.5 Proof of Theorem 1 when idiosyncratic risk grows large}

We next consider a sequence where $\sigma_{I} \rightarrow \infty$. Here the key is to change the "clock," i.e., the speed with which we go through the Brownian sample paths. This effectively reduces both $\sigma_{I}$ and $\sigma_{A}$ at the same rate, converting our sequence of models into one in which $\sigma_{A} \rightarrow 0$. 
Lemma 8 Fix $\tau>0$, and let $\left(\widetilde{a}_{t}^{i}, \widetilde{x}_{t}^{i}\right)$ denote the optimal solution of a model with deep parameters $\tau \cdot\left(\rho, r, \pi, \sigma^{2}, \pi_{I}, \sigma_{i}^{2}\right)$, fixed costs $\bar{\lambda}=\left(\bar{\lambda}_{1}, \bar{\lambda}_{2}\right)$, curvature $\gamma$ and relative preference $\kappa$. Then the process $\left(\widetilde{a}_{t}^{i}, \widetilde{x}_{t}^{i}\right)$ has the same distribution as $\tau \cdot\left(a_{\tau t}^{i}, x_{\tau t}^{i}\right)$ : rescaling the time dimension acts the same way as rescaling the parameters of the model.

Consider a sequence of models where $\sigma_{I} \rightarrow \infty$ and let $\tau=\left(\sigma_{I}\right)^{-2}$. Changing the clock, dynamics will be identical to a model with parameters $\left(\tau \sigma_{I}^{2}, \tau \sigma_{A}^{2}, \tau r, \tau \mu_{A}, \gamma, \bar{\lambda}_{1}, \bar{\lambda}_{2}, \kappa\right)=\left(1, \tau \sigma_{A}^{2}, \tau r, \tau \mu_{A}, \bar{\lambda}_{1}, \bar{\lambda}_{2}, \kappa\right)$. Along this sequence aggregate risk goes to zero while other parameters remain bounded. Hence this model is close to its habit representation; but then so is the original model.

\section{A.6 Proof map for Section 4}

These proofs - which build on the ideas described above - are in the Supplementary Appendix.

\section{Appendix B: Simulations}

Details are in the Supplementary Appendix. Our strategy is to choose deep parameters to generate variation in the consumption risk parameters $\sigma_{I}$ and $\sigma_{A}$ while holding fixed consumption growth. In all environments of Figures 1-3, the parameters $\left(\gamma, \kappa, \lambda_{1}, \lambda_{2}, \delta\right)=(2,1,1,0, .0326)$ are held fixed. Other parameters and the implied values of $\sigma_{A}, \sigma_{I}, \mu_{a}$ and $\mu_{A}$ are given below.

\begin{tabular}{l|lll|llll} 
& $\pi_{M} / \sigma_{M}$ & $\pi_{E} / \sigma_{E}$ & $r$ & $\sigma_{A}$ & $\sigma_{I}$ & $\mu_{a}$ & $\mu_{A}$ \\
\hline (a) High aggr, low idiosyncr risk & $20 \%$ & $10 \%$ & $3.24 \%$ & $10 \%$ & $5 \%$ & $1.24 \%$ & $1.37 \%$ \\
(b) High aggr, high idiosyncr risk & $20 \%$ & $20 \%$ & $1 \%$ & $10 \%$ & $10 \%$ & $.87 \%$ & $1.37 \%$ \\
(c) Low aggr, low idiosyncr risk & $10 \%$ & $10 \%$ & $4.74 \%$ & $5 \%$ & $5 \%$ & $1.24 \%$ & $1.37 \%$ \\
(d) Low aggr, high idiosyncr risk & $10 \%$ & $20 \%$ & $2.5 \%$ & $5 \%$ & $10 \%$ & $.87 \%$ & $1.37 \%$
\end{tabular}




\title{
Supplementary Material to "Consumption Commitments and Habit Formation"
}

\author{
Raj Chetty \\ Adam Szeidl \\ Harvard University and NBER Central European University and CEPR
}

September 2015

This material supplements the paper "Consumption Commitments and Habit Formation. We provide missing proofs for results stated in the main paper and we explain the numerical methods used to simulate the model.

\section{A-1 Proofs of Propositions 2 and 3}

Proof of Proposition 2. Since the only risky assets for household $i$ are $S$ and $S^{i}$, there exists a unique state price density associated with the household-specific private market. The following dynamics for adjustable consumption generates a state price density that prices both risky assets as well as the safe asset

$$
a_{t}^{i}=a_{0}^{i} \exp \left\{\frac{1}{\gamma}\left(\frac{\pi^{2}}{2 \sigma^{2}}+\frac{\pi_{I}^{2}}{2 \sigma_{I}^{2}}+r-\rho\right) t+\frac{\pi}{\gamma \sigma} z_{t}+\frac{\pi_{I}}{\gamma \sigma_{I}} z_{t}^{i}\right\}
$$

and hence must describe the optimal choice of household $i$. Because $a_{0}^{i}=A_{0}$ for all $i$, aggregating across $i$ yields, by the strong law of large numbers for a continuum of agents (Sun, 1998)

$$
\begin{aligned}
A_{t} & =A_{0} \exp \left\{\frac{1}{\gamma}\left(\frac{\pi^{2}}{2 \sigma^{2}}+\frac{\pi_{I}^{2}}{2 \sigma_{I}^{2}}+r-\rho\right) t+\frac{\pi}{\gamma \sigma} z_{t}\right\} \int_{i} \exp \left\{\frac{\pi_{I}}{\gamma \sigma_{I}} z_{t}^{i}\right\} d i \\
& =A_{0} \exp \left\{\frac{1}{\gamma}\left(\frac{\pi^{2}}{2 \sigma^{2}}+\frac{\pi_{I}^{2}}{2 \sigma_{I}^{2}}\left(1+\frac{1}{\gamma}\right)+r-\rho\right) t+\frac{\pi}{\gamma \sigma} z_{t}\right\} .
\end{aligned}
$$

\footnotetext{
${ }^{1}$ E-mails: chetty@fas.harvard.edu, szeidla@ceu.edu.
} 
Define a new discount rate $\delta=\rho-\left(1+\frac{1}{\gamma}\right) \pi_{I}^{2} /\left(2 \sigma_{I}^{2}\right)$. Then the dynamics of aggregate adjustable consumption is given by

$$
A_{t}=A_{0} \exp \left\{\frac{1}{\gamma}\left(\frac{\pi^{2}}{2 \sigma^{2}}+r-\delta\right) t+\frac{\pi}{\gamma \sigma} z_{t}\right\}
$$

This is exactly the dynamics of adjustable consumption that would obtain for a representative consumer with power utility over $A_{t}$ and discount rate $\delta$ who can invest in the publicly traded risky and safe assets.

Proof of Proposition 3. We are interested in characterizing the evolution of the conditional distribution of $y_{t}^{i}$ given a realization of the path of $A$ under $Q$. Using (15) we obtain

$$
d \log a_{t}^{i}=\frac{1}{\gamma}\left(\frac{\pi^{2}}{2 \sigma^{2}}+\frac{\pi_{I}^{2}}{2 \sigma_{I}^{2}}+r-\rho\right) d t+\frac{\pi}{\gamma \sigma} d z+\frac{\pi_{I}}{\gamma \sigma_{I}} d z^{i}=\theta d t+\frac{\pi}{\gamma \sigma} d z+\frac{\pi_{I}}{\gamma \sigma_{I}} d \bar{z}^{i}
$$

where

$$
\theta=\frac{1}{\gamma}\left(\frac{\pi^{2}}{2 \sigma^{2}}+\frac{\pi_{I}^{2}}{2 \sigma_{I}^{2}}+r-\rho\right)+\frac{\pi_{I}^{2}}{\gamma^{2} \sigma_{I}^{2}}
$$

is the drift under $Q$. We first show that $F(y, t)$ is absolutely continuous for all $t>0$ for almost all realizations of the path of aggregate shocks. We do this assuming that the initial condition is $a_{0}^{i}=A_{0}$ and $x_{0}^{i}=X_{0}$ for all agents $i$, i.e., that the initial distribution $F_{0}(y)$ is concentrated on a single point. For other initial distributions the density $f(y, t)$ can simply be computed as an integral of these densities with respect to $F_{0}(y)$.

Throughout the argument we work with the probability measure $Q$. Our proof logic is to fix $t=T$ and the realization of $A_{t}$ for $t \in[0, T]$, pick a collection of intervals $I \subset[L, U]$, compute an upper bound on the probability that $y_{T} \in I$, and then establish that the upper bound goes to zero as the total length of these intervals, denoted $|I|$, goes to zero. Our upper bound is obtained by separately bounding the probabilities of two events.

(1) Reaching $I$ through paths that do not involve "too many" adjustments. Let $\widetilde{y}_{0}^{i}=y_{0}$ and

$$
d \widetilde{y}_{t}^{i}=-\theta \cdot d t-\sigma_{A} \cdot d z_{t}-\sigma_{I} \cdot d \bar{z}_{t}^{i}
$$

Given the dynamics of $\log a_{t}^{i}$, this specification implies that the evolution of $\widetilde{y}$ is the same as that of $y$ except for the discrete adjustments. In particular, $\widetilde{y}_{t}^{i}=y_{t}^{i}$ before the first adjustment occurs. More generally, if $y^{i}$ experiences $n_{U}$ upward and $n_{D}$ downward adjustments in the interval $[0, t]$, 
then $y_{t}^{i}=\widetilde{y}_{t}^{i}+n_{D}(M-L)-n_{U}(U-M)$. Because $\widetilde{y}_{t}^{i}$ is a Brownian motion with a drift, its density is bounded from above by some constant which depends on the parameters of the process, which we denote by $K\left(\mu_{a}, \sigma_{A}, \sigma_{I}, T\right)$. As a result, for any given $n \geq 1$, the total probability of paths which involve $n_{U}<n$ upward and $n_{D}<n$ downward adjustments such that $y_{T}^{i} \in I$ is at most $K\left(\mu_{a}, \sigma_{A}, \sigma_{I}, T\right) \cdot n^{2} \cdot|I|$.

(2) The total probability of paths that involve at least $n$ adjustments. Let $\widetilde{y}_{0}^{A}=y_{0}$ and $d \widetilde{y}_{t}^{A}=-\theta \cdot d t-\sigma_{A} \cdot d z_{t}$ so that $\widetilde{y}_{t}^{A}$ represents the aggregate shocks and trend in $\widetilde{y}_{t}$, and let $\widetilde{y}_{0}^{I, i}=0$ and $d \widetilde{y}_{t}^{I, i}=\sigma_{I} \cdot d \bar{z}_{t}^{i}$ so that $\widetilde{y}_{t}^{I, i}$ represents the idiosyncratic shocks. Then $\widetilde{y}_{t}^{i}=\widetilde{y}_{t}^{A}+\widetilde{y}_{t}^{I, i}$. The path of $\widetilde{y}_{t}^{A}$ contains the same information as the path of aggregate shocks $A_{t}$, hence we are effectively conditioning on the realization of the path of $\widetilde{y}_{t}^{A}$. Set $\Delta_{y}=\min (U-M, M-L) / 2$. We say that a process $u_{t}$ moves $\Delta_{y}$ between $s$ and $t$ if $\left|u_{t}-u_{s}\right|=\Delta_{y}$. Suppose that $s_{1}<s_{2}$ are two consecutive adjustment dates for household $i$. Then either $\widetilde{y}_{t}^{A}$ or $\widetilde{y}_{t}^{I, i}$ must move at least $\Delta_{y}$ between $s_{1}$ and $s_{2}$. Because almost surely the path of $\widetilde{y}_{t}^{A}$ is continuous, one can straightforwardly verify that there is an upper bound $K\left(\widetilde{y}_{[0, T]}^{A}\right)$ on the number of non-overlapping time intervals in $[0, T]$ over which $y_{t}^{A}$ moves at least $\Delta_{y}$. For ease of notation, in the rest of this proof we will simply denote $K\left(\widetilde{y}_{[0, T]}^{A}\right)=K$. Then, if household $i$ adjusts at least $n$ times in $[0, T]$, there must exist at least $n-K$ non-overlapping intervals in $[0, T]$ over which $\widetilde{y}_{t}^{I, i}$ moves at least $\Delta_{y}$. Assume now that $n>2 K+1$. At least one of these intervals - denote it by $\left[s_{1}, s_{2}\right]$-cannot be longer than $T /(n-K)$. Now cover the $[0, T]$ interval with subintervals of length $2 T /(n-K)$ starting at zero, and by another set starting at $T /(n-K)$. It is clear that an interval in one of these covers, say $\left[s_{0}, s_{3}\right]$ must fully contain $\left[s_{1}, s_{2}\right]$.

The probability that $\widetilde{y}_{t}^{I, i}$ moves at least $\Delta_{y}$ over $\left[s_{1}, s_{2}\right]$ is bounded by the probability that the difference between the minimum and the maximum of $\widetilde{y}_{t}^{I, i}$ in $\left[s_{0}, s_{3}\right]$ is at least $\Delta_{y}$. Given that the density of the running maximum of a standard Brownian motion is $(2 /(\pi t))^{1 / 2} e^{-m^{2} /(2 t)}$, this probability is bounded above by a universal constant times $\left((n-K) /\left(\pi T \sigma_{I}^{2}\right)\right)^{1 / 2} \exp \left[-\Delta^{2}(n-K) /\left(2 T \sigma_{I}^{2}\right)\right]$. Because the total number of intervals in the two covers we introduced is at most $2(n-K)$, the probability that $\widetilde{y}_{t}^{I, i}$ moves at least $\Delta_{y}$ over an interval of length at most $T /(n-K)$ is bounded from above by a constant (which depends on $T$ and $\left.\sigma_{I}\right)$ times $(n-K)^{3 / 2} \exp \left[-\Delta^{2}(n-K) /\left(2 T \sigma_{I}^{2}\right)\right]$. Recalling the assumption that $n>2 K+1$, the last expression can be bounded above by a different constant (which depends on $T$ and $\left.\sigma_{I}^{2}\right)$ times $\exp \left[-\Delta^{2} n /\left(8 T \sigma_{I}^{2}\right)\right]$.

We now combine these bounds. Given $K$, which is determined by the path of $\widetilde{y}_{t}^{A}$, and main- 
taining $n>2 K+1$, the total probability that $y_{T}^{i} \in I$ is at most

$$
K\left(\mu_{a}, \sigma_{A}, \sigma_{I}, T\right) \cdot n^{2} \cdot|I|+K\left(\sigma_{I}^{2}, T\right) \cdot \exp \left[-\Delta^{2} n /\left(8 T \sigma_{I}^{2}\right)\right]
$$

Setting $n=|I|^{-1 / 4}$, for small enough $|I|$ such that $n>2 K+1$ is satisfied, the bound becomes

$$
K\left(\mu_{a}, \sigma_{A}, \sigma_{I}, T\right) \cdot|I|^{1 / 2}+K\left(\sigma_{I}^{2}, T\right) \cdot \exp \left[-\Delta^{2}|I|^{-1 / 4} /\left(8 T \sigma_{I}^{2}\right)\right]
$$

which goes to zero as $|I|$ goes to zero.

We now turn to the stochastic partial differential equation. Proposition 1 in Caballero (1993) derives a stochastic partial differential equation, given the path of aggregate shocks, for the conditional density of a double-barrier Brownian motion with rebirth. Caballero's equation is

$$
d f(y, t)=\left[\theta \frac{\partial f(y, t)}{\partial y}+\frac{\sigma_{T}^{2}}{2} \frac{\partial^{2} f(y, t)}{\partial y^{2}}\right] d t+\sigma_{A} \frac{\partial f(y, t)}{\partial y} d z
$$

Substituting in (16) yields the equation in the text. The boundary conditions follow directly from Caballero's proposition.

To derive the dynamics of aggregate commitments, note that $X_{t}=\int_{L}^{U} e^{y} f(y, t) d y \cdot A_{t}$ and we can use Ito's lemma to write

$$
d X_{t}=A_{t} \int_{L}^{U} e^{y} \cdot d f(y, t) \cdot d y+d A_{t} \cdot \int_{L}^{U} e^{y} f(y, t) d y+\left\langle\int_{L}^{U} e^{y} \cdot d f(y, t) \cdot d y, d A_{t}\right\rangle
$$

We now evaluate each term on the right hand side. The first term is

$$
A_{t} \int_{L}^{U} e^{y} \cdot \frac{\partial f(y, t)}{\partial y}\left\{\left(\mu+\frac{\pi_{I}^{2}}{2 \gamma^{2} \sigma_{I}^{2}}\right) d t+\frac{\pi}{\gamma \sigma} d z\right\} d y+F_{t} \int_{L}^{U} e^{y} \cdot \frac{\partial^{2} f(y, t)}{\partial y^{2}} \frac{\sigma_{T}^{2}}{2} d t \cdot d y
$$

Integrating by parts, and using the boundary conditions shows that this term equals

$$
-X_{t}\left(\left(\mu+\frac{\pi_{I}^{2}}{2 \gamma^{2} \sigma_{I}^{2}}\right) d t+\frac{\pi}{\gamma \sigma} d z\right)+A_{t} \frac{\sigma_{T}^{2}}{2} \cdot\left(f_{y}(L, t)\left(e^{M}-e^{L}\right)+f_{y}(U, t)\left(e^{U}-e^{M}\right)\right) d t+\frac{\sigma_{T}^{2}}{2} X_{t} d t .
$$

The second term is

$$
X_{t} \cdot \frac{d A_{t}}{A_{t}}=X_{t}\left(\left(\mu+\frac{\pi^{2}}{2 \gamma^{2} \sigma^{2}}\right) d t+\frac{\pi}{\gamma \sigma} d z\right)
$$

while the third term is simply $-\pi^{2} /(\gamma \sigma)^{2} X_{t} d t$. Collecting terms gives the result of the proposition. 


\section{A-2 Proofs of results leading up to Theorem 1}

\section{A-2.1 Proofs of auxiliary results about the commitments model including proof of Proposition 4}

Proof of Lemma 1. We start with the case where $w_{t}$ is driven by a standard Brownian motion. Let $\zeta_{y}=\inf \left\{t \geq 0: w_{t} \notin[L, U], w_{0}=y\right\}$. Set $F_{w}(t)=\operatorname{Pr}\left[\zeta_{y} \leq t\right]$ and $\bar{h}(y, t)=E\left[e^{w_{t}} \cdot 1\left\{\zeta_{y}>t\right\}\right]$ be $h(y, t)$ killed at the boundary. Let $F_{y}^{(1)}(t)=F_{y}(t)$ and $F_{y}^{(n+1)}(t)=\int_{0}^{t} F_{y^{*}}^{(n)}(t-\tau) d F_{y}(\tau)=$ $\int_{0}^{t} F_{M}(t-\tau) d F_{y}^{(n)}(\tau)$ be the the distribution of the $n+1$ st exit time. Then

$$
h(y, t)=\bar{h}(y, t)+\sum_{n=1}^{\infty} \int_{0}^{t} \bar{h}(M, t-\tau) d F_{y}^{(n)}(\tau)=\bar{h}(y, t)+\int_{0}^{t} \bar{h}(M, t-\tau) d F_{y}^{*}(\tau)
$$

where

$$
F_{y}^{*}(t)=\sum_{n=1}^{\infty} F_{y}^{(n)}(t)=F_{y}(t)+\int_{0}^{t} F_{M}^{*}(t-\tau) d F_{y}(\tau)=F_{y}(t)+\int_{0}^{t} F_{M}(t-\tau) d F_{y}^{*}(\tau)
$$

is the expected number of boundary hits until $t$.

The transition density of the killed diffusion $p\left(y, y^{\prime}, t\right)=\operatorname{Pr}\left[\zeta_{y}>t, y_{t}=y^{\prime}\right]$ can be expressed as an infinite sum of normal densities (Revuz and Yor, 1992, p 106), and in particular, is infinitely many times differentiable in $[L, U] \times[L, U] \times(0, \infty)$. This implies that $\bar{h}(y, t)=\int e^{y^{\prime}} p\left(y, y^{\prime}, t\right) d y^{\prime}$ is infinitely many times differentiable in $[L, U] \times(0, \infty)$. The density of the first hitting time $\zeta_{y}$ can also be expressed in closed form as an infinite sum (Darling and Sieger, 1953), and is infinitely many times differentiable in $y$ and $t$ over $[L, U] \times(0, \infty)$. This, combined with (18) implies that $F_{y}^{*}(t)$ is $C^{\infty}$ in $[L, U] \times(0, \infty)$. Combining these observations with $(17)$ shows that $h(y, t)$ is also $C^{\infty}$ in the $[L, U] \times(0, \infty)$ domain. $^{1}$

We next show that $h$ is also smooth when driven by any Brownian motion with drift and variance, and that it is smooth in the other parameters. Changing the clock of $y_{t}$ scales both the mean and the variance, and is obviously a smooth transformation of $h(y, t)$ as it just scales the time argument. Shifting and rescaling the vertical axis are smooth operations that shift and rescale the triple $[L, M, U]$. Thus we only need to show smoothness in the drift and in $M$. The drift can be dealt with using the Girsanov theorem, which implies that the density of the killed diffusion under drift can be obtained as $p^{\mu w}\left(y, y^{\prime}, t\right)=p\left(y, y^{\prime}, t\right) \cdot \exp \left[\mu_{w}\left(y^{\prime}-y\right)-\mu_{w}^{2} t / 2\right]$, which

\footnotetext{
${ }^{1}$ Grigorescu and Kang (2002) compute the transition density of $y$ explicitly.
} 
is clearly $C^{\infty}$ in $\mu_{w}$, and hence so is $\bar{h}(y, t)$. Next, the distribution of the first hitting time is $1-F_{y}^{\mu_{y}}(t)=\int p^{\mu_{y}}\left(y, y^{\prime}, t\right) d y^{\prime}$ is also smooth. The smoothness of $h$ in $\mu_{y}$ now follows from (17). Smoothness in $M$ follows easily from (17).

Proof of Lemma 2. We have

$$
E_{s}\left[\bar{X}_{t}\right]=\bar{A}_{s} \cdot E_{s}^{R}\left[\bar{X}_{t} / \bar{A}_{t}\right]=\bar{A}_{s} \cdot E_{s}^{Q R}\left[x_{t} / a_{t}\right]=\bar{A}_{s} \cdot \int_{L}^{U} h(t-s, y) f(y, s) d y
$$

which is a martingale in $s$. Computing the Ito-differential

$$
d_{s} E_{s}\left[\bar{X}_{t}\right]=d \bar{A}_{s} \cdot E_{s}^{Q R}\left[x_{t} / a_{t}\right]+\bar{A}_{s} \cdot \int_{L}^{U} h(t-s, y) f_{y}(y, s) \sigma_{A} d z_{s} \cdot d y
$$

where we used (7) for the evolution of $f(y, s)$ and collected only the $d z$ terms, since the $d s$ terms must cancel by the martingale property. Equivalently,

$d_{s} E_{s}\left[\bar{X}_{t}\right]=d \bar{A}_{s} \cdot\left(E_{s}^{Q R}\left[x_{t} / a_{t}\right]+\int_{L}^{U} h(t-s, y) f_{y}(y, s) d y\right)=d \bar{A}_{s} \cdot \int_{L}^{U}\left(h(u, y)-h_{y}(u, y)\right) f(y, s) d y$

where we integrated by parts. This equation shows the existence of $\xi$ as well as the desired representation.

Proof of Lemma 3. Ben-Ari and Pinsky (2009) show that $y_{t}=\log \left[x_{t} / a_{t}\right]$ converges exponentially fast to a unique invariant distribution. it follows from Ben-Ari and Pinsky (2007) that the rate of convergence is uniformly bounded if the drift is from a bounded interval. This implies uniform convergence for all $\sigma_{A} \in\left[0, \bar{\sigma}_{A}\right]$ through a clock-change argument. Since

$$
E_{0}\left[\bar{X}_{t}\right]=E_{0}^{R}\left[\bar{X}_{t} / \bar{A}_{t}\right]=E_{0}^{Q R}\left[x_{t} / a_{t}\right]
$$

it follows that $E_{0}\left[\bar{X}_{t}\right]$ converges exponentially fast to the mean $\bar{x}$ of $x / a$ under the invariant distribution, and that this is uniform in $\sigma_{A}$. Recalling that $h(u, y)=E^{Q R}\left[x_{u} / a_{u} \mid x_{0} / a_{0}=e^{y}\right]$, we also have $h(u, y)$ converge at the same rate to $\bar{x}$ as $u \rightarrow \infty$, uniformly in $y$ and $\sigma_{A}$. Letting $F_{t}^{Q R}\left[y \mid y_{0}\right]$ denote the cross-sectional distribution of $y_{t}$ given initial value $y_{0}$, fixing some $s<u$, we 
can write

$$
\begin{aligned}
h_{y_{0}}\left(u, y_{0}\right) & =\frac{\partial}{\partial y_{0}} \int_{L}^{U} h(u-s, y) d F_{t}^{Q R}\left[y \mid y_{0}\right]=\int_{L}^{U} h(u-s, y) \frac{\partial^{2} F_{t}^{Q R}\left[y \mid y_{0}\right]}{\partial y_{0} \partial y} d y \\
& =\int_{L}^{U}(h(u-s, y)-\bar{x}) \frac{\partial^{2} F_{t}^{Q R}\left[y \mid y_{0}\right]}{\partial y_{0} \partial y} d y
\end{aligned}
$$

where at the last step we used that $\partial^{2} F_{t}^{Q R}\left[y \mid y_{0}\right] / \partial y_{0} \partial y$ integrates to zero in $y$. By the arguments of Lemma $1, \partial^{2} F_{t}^{Q R}\left[y \mid y_{0}\right] / \partial y_{0} \partial y$ is bounded, while $h(u-s, y)-\bar{x}$ converges exponentially fast to zero; hence so does the integral.

Proof of Proposition 4. We show that $\xi(u, f)$ equals the impulse response of Definition 1. Let $\bar{A}_{0}^{*}$ be the point at which we want to differentiate $E_{0}\left[\bar{X}_{t}\left(A_{0}, F^{x}\left(x_{0} \mid A_{0}^{*}\right)\right)\right]$. We can write

$$
\begin{aligned}
E_{0}\left[\bar{X}_{t}\left(A_{0}, F^{x}\left(x_{0} \mid A_{0}^{*}\right)\right)\right] & =A_{0} \cdot E_{0}^{R}\left[\bar{X}_{t}\left(A_{0}, F^{x}\left(x_{0} \mid A_{0}^{*}\right)\right)_{t} / \bar{A}_{t}\right] \\
& =A_{0} \cdot \int_{L}^{U} h\left(t, y-\left(\log A_{0}-\log A_{0}^{*}\right)\right) d F_{0}(y) .
\end{aligned}
$$

This is because when $\bar{A}_{0}=\bar{A}_{0}^{*}$, the mass of people at any point $y$ is given by $d F_{0}(y)$, and the conditional expectation given $y$ is summarized by $h$. When $\bar{A}_{0}$ changes, the mass of these people is unaffected, and hence $d F_{0}(y)$ is unchanged; but-because commitments are held fixed while $A_{0}$ changes - their $y$ shifts. Hence we must evaluate $h$ at a point which recognizes this change.

Differentiating this expression in $A_{0}$ gives

$\frac{E_{0}\left[\bar{X}_{t}\left(A_{0}, F^{x}\left(x_{0} \mid A_{0}^{*}\right)\right)\right]}{\partial A_{0}}=\int_{L}^{U} h(t, y) d F_{0}(y)-\int_{L}^{U} h_{y}(t, y) d F_{0}(y)=\int_{L}^{U}\left[h(t, y)-h_{y}(t, y)\right] d F_{0}(y)$

which is exactly the definition of $\xi$ given above when $F_{0}(y)$ has a density. This confirms that the impulse response is well defined, that it is independent of $A_{0}^{*}$, and that the MA representation claimed in the proposition holds.

Proof of Lemma 4. We know that EF converges to $F^{*}$ uniformly in $y$. Fix $\varepsilon>0$ and pick $s$ so that for all $t>s,\left|E F_{t}-F^{*}\right|<\varepsilon / 8$ for all initial conditions and for all $\sigma$ small enough. Consider the rectangular set $[-\kappa, \kappa] \times[t-s, t]$, and let $G_{\kappa}$ denote the event when the realization of $\log \bar{A}_{u}-\log \bar{A}_{t-s}$ for $u \in[t-s, t]$ is in this set. Let $F\left(y, t, \bar{A}_{[t-s, t]}, y_{s}\right)$ denote the distribution of $y_{t}$ under $Q$ when started at $y_{s}$ in $s$, and when the realization of aggregate shocks is given by $\bar{A}_{[t-s, t]}$. We then have that $\left\{\sup _{y_{t}, y_{s}}\left|F\left(y, t, \bar{A}_{[t-s, t]}, y_{s}\right)-F\left(y, t, \bar{A}_{[t-s, t]}^{\prime}, y_{s}\right)\right| \bar{A}_{[t-s, t]}, \bar{A}_{[t-s, t]}^{\prime} \in G_{\kappa}\right\}$ goes 
to zero as $\kappa \rightarrow 0$ : two sufficiently close paths of aggregate consumption generate cross-sectional distributions that are themselves close. This is because the share of people for whom the two aggregate paths result in sufficiently different behavior goes to zero. Take $\kappa$ small enough so that this quantity is less than $\varepsilon / 8$. For any fixed $\kappa$ we can pick $\sigma$ small enough so that $\operatorname{Pr}\left[\bar{A}_{[t-s, t]} \in G_{\kappa}\right]>$ $1-\varepsilon / 8$. This implies that $\left|E_{s} F_{t}-E\left[F_{t} \mid f(s), G_{\kappa}\right]\right|<\varepsilon / 4$. Combining these bounds, for $\bar{A}_{[t-s, t]} \in$ $G_{\kappa}$ we have

$$
\begin{aligned}
& \quad\left|F\left(y, t, \bar{A}_{[t-s, t]}, f(s)\right)-F^{*}(y)\right| \leq \\
& \left|F\left(y, t, \bar{A}_{[t-s, t]}, f(s)\right)-E\left[F_{t} \mid f(s), G_{\kappa}\right]\right|+\left|E\left[F_{t} \mid f(s), G_{\kappa}\right]-E_{s} F_{t}\right|+\left|E_{s} F_{t}-F^{*}(y)\right|<\frac{\varepsilon}{8}+\frac{\varepsilon}{4}+\frac{\varepsilon}{8}=\frac{\varepsilon}{2} .
\end{aligned}
$$

Using this, we have

$$
\begin{aligned}
&\left\|\sup _{y}\left|F(y, t)-F^{*}(y)\right|\right\|_{p}^{p}= \\
& \operatorname{Pr}\left[G_{\kappa}\right] \cdot E\left[\sup _{y}\left(F(y, t)-F^{*}(y)\right)^{p} \mid G_{\kappa}\right]+\left(1-\operatorname{Pr}\left[G_{\kappa}\right]\right) \cdot E\left[\sup _{y}\left(F(y, t)-F^{*}(y)\right)^{p} \mid \operatorname{not} G_{\kappa}\right] \leq \\
& {\left[\left(\frac{\varepsilon}{2}\right)^{p}+2^{p} \frac{\varepsilon}{8}\right]<2^{p} \varepsilon . }
\end{aligned}
$$

Since this is true for all $t>s$, it is also true for the lim sup. But $\varepsilon$ was arbitrary, and the bound applies for all $\sigma$ small enough given $\varepsilon$; hence the desired result follows.

\section{A-2.2 Proofs of auxiliary results about ther habit model}

Proof of Lemma 5. Starting with the $A$-weighted habit model, consider the unique solution of the integral equations for $\zeta$ and $o$ (see Lew, 1972 for existence and uniqueness) and define

$$
\widetilde{X}_{t}=o(t) X_{0}+\int_{0}^{t} \zeta(t-s) C_{s} d s
$$

We will show that $\widetilde{X}_{t}=X_{t}$ for all $t \geq 0$. First note that

$$
\begin{aligned}
\widetilde{X}_{t} & =o(t) X_{0}+\int_{0}^{t} \zeta(t-s)\left[A_{s}+X_{s}\right] d s \\
& =o(t) X_{0}+\int_{0}^{t} \zeta(t-s) A_{s}+\zeta(t-s)\left[\int_{0}^{s} j(s-u) A_{u} d u+k(s) X_{0}\right] d s \\
& =o(t) X_{0}+\int_{0}^{t} A_{s}\left[\zeta(t-s)+\int_{0}^{t-s} j(u) \zeta(t-s-u) d u\right] d s+X_{0} \int_{0}^{t} \zeta(t-s) k(s) d s .
\end{aligned}
$$


Equating coefficients, $X_{t}=\widetilde{X}_{t}$ holds if

$$
j(t-s)=\zeta(t-s)+\int_{0}^{t-s} j(u) \zeta(t-s-u) d u
$$

or, with $t-s=u$,

$$
\zeta(u)=j(u)-\int_{0}^{u} \zeta(v) j(u-v) d v
$$

and

$$
o(u)=k(u)-\int_{0}^{u} \zeta(u-v) k(v) d v .
$$

Substituting in $u=0$ gives $\zeta(0)=j(0)$ and $o(0)=k(0)$. The integral equation for $\zeta(u)$ then yields a unique solution, which can be used to determine $o($.$) . By the above argument, a pair of functions$ that solve these equations also give $X_{t}=\widetilde{X}_{t}$, which is the desired representation.

Proof of Lemma 6. Detrending both sides and integrating by parts (using that $\xi^{*}$ is smooth)

$$
\begin{aligned}
\bar{X}_{t}^{h} & =\int_{0}^{t} \xi^{* \prime}(t-s) \bar{A}_{s} d s+\left[\bar{x}-\xi^{*}(t)\right] A_{0}=\left[-\xi^{*}(t-u) \bar{A}_{u}\right]_{0}^{t}+\int_{0}^{t} \xi^{*}(t-s) d \bar{A}_{s}+\left[\bar{x}-\xi^{*}(t)\right] A_{0} \\
& =\int_{0}^{t} \xi^{*}(t-s) d \bar{A}_{s}+\bar{x} A_{0} .
\end{aligned}
$$

\section{A-2.3 Proofs of results used in establishing Theorem 1}

Proof of Lemma \%. We proceed by induction on $t$. Fix some $k>0$. We show that (i) the desired bound holds when $t \leq k$, and (ii) if the bound holds for some $t$, it also holds for $t+k$. We begin by showing (ii), which is the more difficult part.

We can write

$$
\left\|G_{t}\right\|_{p} \leq\left\|\frac{\bar{A}_{t-k}}{\bar{A}_{t}} \int_{t-k}^{t} g(t-s) \frac{\bar{A}_{s}}{\bar{A}_{u-k}} d z_{s}\right\|_{p}+\left\|\frac{\bar{A}_{t-k}}{\bar{A}_{t}}\right\|_{p} \cdot\left\|\frac{1}{\bar{A}_{t-k}} \int_{0}^{t-k} g(t-s) \bar{A}_{s} d z_{s}\right\|_{p}
$$

where we used independence of the Brownian increments. Denoting $\bar{g}(u, s)=e^{K_{2} k} g(u+k, s)$ we can rewrite the final term in brackets as

$$
e^{-K_{2} k} \cdot \frac{1}{\bar{A}_{t-k}} \int_{0}^{t-k} \bar{g}(t-k-s, s) A_{s} d z_{s}
$$

where $|\bar{g}(u, s)| \leq K_{1} e^{-K_{2} u}$ by construction. By our induction assumption, this term has $p$-norm 
bounded by $e^{-K_{2} k} \cdot M(p)$. To bound the remaining terms, first observe that by lognormality

$$
\left\|\frac{\bar{A}_{t-k}}{\bar{A}_{t}}\right\|_{p} \leq K_{p}\left(\sigma_{A}, k\right)
$$

for some $K_{p}\left(\sigma_{A}, k\right)$ that goes to one in $\sigma_{A}$ for all $k$. Next note that

$$
\left\|\frac{\bar{A}_{t-k}}{\bar{A}_{t}} \int_{t-k}^{t} g(t-s, s) \frac{\bar{A}_{s}}{\bar{A}_{t-k}} d z_{s}\right\|_{p} \leq\left\|\frac{\bar{A}_{t-k}}{\bar{A}_{t}}\right\|_{2 p} \cdot\left\|\int_{t-k}^{t} g(t-s, s) \frac{\bar{A}_{s}}{\bar{A}_{t-k}} d z_{s}\right\|_{2 p}
$$

by the Cauchy-Schwarz inequality. Here

$$
\left\|\frac{\bar{A}_{t-k}}{\bar{A}_{t}}\right\|_{2 p} \leq K_{2 p}\left(\sigma_{A}, k\right)
$$

where $K_{2 p}\left(\sigma_{A}, k\right)$ also goes to one in $\sigma_{A}$ for all $k$. Finally, using standard bounds (e.,g., Karatzas and Shreve, 2008) for moments of the Ito integral, we obtain

$$
\left\|\int_{t-k}^{t} g(t-s, s) \frac{\bar{A}_{s}}{\bar{A}_{t-k}} d z_{s}\right\|_{2 p} \leq K_{2 p}\left(\int_{t-k}^{t} K_{1}^{2}\left\|\left(\frac{\bar{A}_{s}}{\bar{A}_{u-k}}\right)^{2}\right\|_{p} d s\right)^{1 / 2}
$$

which is bounded by $K_{2 p} K_{1} k \cdot K_{2 p}\left(\sigma_{A}, k\right)$. Combining terms we obtain

$$
\left\|G_{t}\right\|_{p} \leq K_{2 p}^{2}\left(\sigma_{A}, k\right) \cdot K_{2 p} K_{1} k+K_{p}\left(\sigma_{A}, k\right) \cdot e^{-K_{2} k} \cdot M(p)
$$

It is easy to see that if

$$
M(p)=\frac{K_{2 p}^{2}\left(\sigma_{A}, k\right) \cdot K_{2 p} K_{1} k}{1-K_{p}\left(\sigma_{A}, k\right) \cdot e^{-K_{2} k}}
$$

is positive, then the induction step follows. We can make sure that this is the case by first choosing some $k>0$, and then picking $\overline{\sigma_{A}}$ small enough so that for all $\sigma_{A} \leq \bar{\sigma}_{A}$ we have $K_{p}\left(\sigma_{A}, k\right)<e^{K_{2} k / 2}$. With this choice of $M(p)$, the induction step follows; and (i) can be verified easily from the argument of the induction step.

Proof of Lemma 8. We verify directly that changing the clock is equivalent to rescaling the relevant parameters in the setup of the problem. Maximizing the consumer's problem in the original model is equivalent to maximizing

$$
E \int_{0}^{\infty} e^{-\rho t \tau}\left(\frac{a_{\tau t}^{1-\gamma}}{1-\gamma}+\mu \frac{x_{\tau t}^{1-\gamma}}{1-\gamma}\right) d t
$$


which is proportional to the objective function in the model with new parameters. Similarly, the budget constraint of the original model implies

$$
d w_{\tau t}=\left[\left(\tau r+\alpha_{\tau t} \tau \pi+\alpha_{\tau t}^{i} \tau \pi_{I}\right) w_{t}-\tau c_{t}\right] d t+\alpha_{\tau t} w_{\tau t} \sigma \tau^{1 / 2} d z_{\tau t}+\alpha_{\tau t}^{i} w_{\tau t} \sigma_{i} \tau^{1 / 2} d z_{\tau t}^{i}
$$

on all non-adjustment dates due to the scaling invariance of Brownian motion. Finally, on adjustment dates, $d w=\bar{\lambda}_{1} x_{t-} / r+\bar{\lambda}_{2} x_{t} / r=\bar{\lambda}_{1} \cdot \tau x_{t-} /(\tau r)+\bar{\lambda}_{2} \cdot \tau x_{t} /(\tau r)$. Since the optimal policy is unique, the claim follows.

\section{A-3 Proofs for Section 4.1}

\section{A-3.1 Proof of Proposition 5}

(1) Excess smoothness. Using a Taylor expression we can write

$$
\log C_{t_{1}}-\log C_{t_{0}}=\frac{A_{t_{0}}}{C_{t_{0}}}\left(\log A_{t_{1}}-\log A_{t_{0}}\right)+\varepsilon_{t_{1}}
$$

where, because $X_{t}$ has bounded variation, there exists $K_{\varepsilon}$ such that $E \varepsilon_{t_{1}}^{2}<K_{e}\left(t_{1}-t_{0}\right)^{2}$. Thus

$\beta_{1}\left(t_{1}\right)=\frac{\operatorname{cov}\left(\log \left(C_{t_{1}} / C_{t_{0}}\right), \log \left(A_{t_{1}} / A_{t_{0}}\right)\right)}{\operatorname{var}\left(\log \left(A_{t_{1}} / A_{t_{0}}\right)\right)} \leq \frac{A_{t_{0}}}{C_{t_{0}}}+\frac{\sigma_{A}\left(t_{1}-t_{0}\right)^{1 / 2} K_{\varepsilon}\left(t_{1}-t_{0}\right)}{\sigma_{A}^{2}\left(t_{1}-t_{0}\right)}=\frac{A_{t_{0}}}{C_{t_{0}}}+\frac{\left(t_{1}-t_{0}\right)^{1 / 2} K_{\varepsilon}}{\sigma_{A}}$

and the right-hand side approaches $A_{t_{0}} / C_{t_{0}}$ as $t_{1} \rightarrow t_{0}$.

(2) Excess sensitivity. Let $t_{1}=t_{2}$. From the proof of Lemma 3 we know that $\log \left[A_{t_{3}} / C_{t_{3}}\right]$ converges exponentially fast to an invariant distribution. In particular, $E_{t_{1}}\left[\log \left[A_{t_{3}} / C_{t_{3}}\right]\right]$ converges exponentially fast to the mean of this invariant distribution, which we denote by $\bar{\xi}$, so that we can write $\log C_{t_{3}}=\log A_{t_{3}}+\bar{\xi}+\varepsilon_{t_{3}}$ where $E_{t_{1}}\left[\varepsilon_{t_{3}}\right]$ converges to zero at a given exponential rate as $t_{3} \rightarrow \infty$. Using (19) we can write

$$
\begin{aligned}
\log C_{t_{3}}-\log C_{t_{1}} & =\log A_{t_{3}}+\bar{\xi}+\varepsilon_{t_{3}}-\log C_{t_{0}}-\frac{A_{t_{0}}}{C_{t_{0}}}\left(\log A_{t_{1}}-\log A_{t_{0}}\right)-\varepsilon_{t_{1}} \\
& =\frac{X_{t_{0}}}{C_{t_{0}}}\left(\log A_{t_{1}}-\log A_{t_{0}}\right)+\left(\log A_{t_{3}}-\log A_{t_{1}}\right)+\left(\log A_{t_{0}}+\bar{\xi}\right)+\left(\varepsilon_{t_{3}}-\varepsilon_{t_{1}}\right) .
\end{aligned}
$$

To compute $\beta_{2}$, we evaluate the covariance of $\log A_{t_{1}}-\log A_{t_{0}}$ with each of the terms in this expression. Because $\log A_{t}$ is a Brownian motion with drift, the covariance with the term in the second parenthesis is zero. Conditional on the history up to $t_{0}$, the terms in the third parenthesis 
are constants, hence their covariance is also zero. The terms in the fourth parenthesis are error terms: just like in the proof of (1), $\varepsilon_{1}$ can be made arbitrarily small by choosing $t_{1}$ small; and $\varepsilon_{t_{3}}$ is approximately orthogonal to events before $t_{1}$ for $t_{3}$ large. Thus for $t_{1}$ small and $t_{3}$ large the regression coefficent is determined by the first term, implying that $\beta_{2}$ is approximately $X_{t_{0}} / C_{t_{0}}>0$.

\section{A-3.2 Modeling large shocks}

Our approach is to construct, on a single probability space, a set of "shock" processes for each $t_{1}>t_{0}$, such that the distribution of the process for a given $t_{1}$ is identical to the distribution of $\bar{A}_{t}$ conditional on the shock event $S\left(t_{1}, \Delta\right)$. This construct will allow us to take limits while holding fixed the probability space.

Formally, we introduce the auxiliary process $\widetilde{A}_{t}$, which agrees with $\bar{A}_{t}$ for $t \leq t_{0}$, and has the same distribution as $\bar{A}_{t}$ for $t>t_{0}$. The idea is that innovations in $\widetilde{A}_{t}$ will be driving $\bar{A}_{t}$ after the shock. We also introduce an independent standard Brownian motion $\bar{B}_{s}$ defined for $s \geq 0$, which will drive the innovations during the shock. We then model the positive shock as a Brownian bridge for $\log \bar{A}_{t}$ conditioned to start at $\log \bar{A}_{t_{0}}$ at time $t_{0}$, and to reach $\log \bar{A}_{t_{0}}+\Delta$ at time $t_{1}$. We denote this process by $\bar{A}_{t}\left(+, t_{1}, \Delta\right)$, and construct it as follows: for $t_{0} \leq t \leq t_{1}$, we let $\log$ $\bar{A}_{t}\left(+, t_{1}, \Delta\right)=\sigma_{A}\left(\bar{B}_{t-t_{0}}-\left(t-t_{0}\right) \bar{B}_{t_{1}}\right)+\left(t-t_{0}\right) \Delta$, and for $t \geq t_{1}$ we let $d \log \bar{A}_{t}\left(+, t_{1}, \Delta\right)=$ $d \log \widetilde{A}_{t}$. Although the expression for $t_{0} \leq t \leq t_{1}$ does not make this clear, it is well-known that this Brownian bridge is an Ito-processes. We construct $\bar{A}_{t}\left(-, t_{1}, \Delta\right)$ analogously. Given that it is a Brownian bridge between $t_{0} \leq t \leq t_{1}$ it follows that $\log \bar{A}_{t}\left(+, t_{1}, \Delta\right)$ has the same distribution as our original process $\log \bar{A}_{t}$ conditional on $S\left(+, t_{1}, \Delta\right)$.

The formulas for the dynamics of $X_{t}, C_{t}, X_{t}^{h}$ and $C_{t}^{h}$, once we replace $\bar{A}_{t}$ by $\bar{A}_{t}\left(+, t_{1}, \Delta\right)$ respectively $\bar{A}_{t}\left(-, t_{1}, \Delta\right)$, directly extend, and generate the distributions of commitments, habit, and consumption conditional on the shock event. To clarify which process we have in mind, we sometimes use notation such as $\bar{X}_{t}\left(+, t_{1}, \Delta\right)$ to refer to aggregate commitments (during or after a positive shock) on the probability space just constructed. However, when it does not cause confusion we often just write $\bar{X}_{t}^{h}$ and say in words that we work with the "shock" processes.

One key feature of this construction is that instead of considering a sequence of non-overlapping events $S\left(t_{1}, \Delta\right)$, we consider a single probability space and a sequence of processes. The advantage is that we can use the $L_{p}$ norm on this common probability space when we take various limits over $\bar{t}$. In particular, throughout the analysis below, we use $L_{p}$ (conditional on the history up to $t_{0}$ ) for all $p \geq 1$ as we take the limits $t_{1} \rightarrow t_{0}$ and $t_{2} \rightarrow t_{0}$. 


\section{A-3.3 Continuity after large shocks}

We show that $\bar{X}_{t}^{h}$ and $\bar{C}_{t}^{h}$ change continuously around $t_{0}$ in the limit as $t_{1} \rightarrow t_{0}$ and as $t_{2} \rightarrow t_{0}$.

Lemma 9 We have $\lim _{t_{1} \rightarrow t_{0}} \bar{X}_{t_{1}}^{h}\left(+, t_{1}, \Delta\right)=\bar{X}_{t_{0}}^{h}$ and $\lim _{t_{1} \rightarrow t_{0}} \bar{X}_{t_{1}}^{h}\left(-, t_{1}, \Delta\right)=\bar{X}_{t_{0}}^{h}$. Moreover, even after taking the limit $t_{1} \rightarrow t_{0}$ the the dynamics of $\bar{X}_{t}^{h}$ continuous at $t_{0}: \lim _{t_{2} \rightarrow t_{0}} \lim _{t_{1} \rightarrow t_{0}} \bar{X}_{t_{2}}^{h}\left(+, t_{1}, \Delta\right)=$ $\bar{X}_{t_{0}}^{h}$ and $\lim _{t_{2} \rightarrow t_{0}} \lim _{t_{1} \rightarrow t_{0}} \bar{X}_{t_{2}}^{h}\left(-, t_{1}, \Delta\right)=\bar{X}_{t_{0}}^{h}$.

Proof. Consider the case when the shock is positive. Suppressing in notation that we work with the "shock" processes, according to the representation in Lemma $7, \bar{X}_{t_{2}}^{h}=\int_{0}^{t_{2}} \xi^{* \prime}\left(t_{2}-s\right) \bar{A}_{s}\left(+, t_{1}, \Delta\right) d s+$ $\left[\bar{x}-\xi^{*}\left(t_{2}\right)\right] A_{0}$. When $t_{2}=t_{1}$ goes to $t_{0}$, this expression converges to $\int_{0}^{t_{0}} \xi^{* \prime}\left(t_{2}-s\right) \bar{A}_{s}\left(+, t_{1}, \Delta\right) d s+$ $\left[\bar{x}-\xi^{*}\left(t_{2}\right)\right] A_{0}=\bar{X}_{t_{0}}^{h}$ proving, for a positive shock, the first claim. For the second claim, note that as $t_{1} \rightarrow t_{0}$ the last term is constant while the first term converges to $\int_{t_{0}}^{t_{2}} \xi^{* \prime}\left(t_{2}-s\right) \widetilde{A}_{s} \cdot e^{\Delta} d s+$ $\int_{0}^{t_{0}} \xi^{* \prime}\left(t_{2}-s\right) \bar{A}_{s} d s$. Here only the first integral depends on $t_{2}$ and as $t_{2} \rightarrow t_{0}$ it converges to zero. The same logic works when the shock is negative.

Lemma 10 We have $\lim _{t_{1} \rightarrow t_{0}} \log \left[\bar{C}_{t_{1}}^{h}\left(+, t_{1}, \Delta\right)\right]=\log \left[e^{\Delta \bar{A}_{t_{0}}}+\bar{X}_{t_{0}}^{h}\right]$ and $\lim _{t_{1} \rightarrow t_{0}} \log \left[\bar{C}_{t_{1}}^{h}\left(-, t_{1}, \Delta\right)\right]=$ $\log \left[e^{-\Delta} \bar{A}_{t_{0}}+\bar{X}_{t_{0}}^{h}\right]$. And analogously we have $\lim _{t_{2} \rightarrow t_{0}} \lim _{t_{1} \rightarrow t_{0}} \log \left[\bar{C}_{t_{2}}^{h}\left(+, t_{1}, \Delta\right)\right]=\log \left[e^{\Delta} \bar{A}_{t_{0}}+\bar{X}_{t_{0}}^{h}\right]$ and $\lim _{t_{2} \rightarrow t_{0}} \lim _{t_{1} \rightarrow t_{0}} \log \left[\bar{C}_{t_{2}}^{h}\left(-, t_{1}, \Delta\right)\right]=\log \left[e^{-\Delta} \bar{A}_{t_{0}}+\bar{X}_{t_{0}}^{h}\right]$.

Proof. Suppose the shock is positive. Then, supressing in notation that we work with the "shock" processes, using the fact that $\log (1+z) \leq z$,

$$
\begin{aligned}
& \left|\log \left[\bar{C}_{t_{2}}^{h}\right]-\log \left[e^{\Delta} \bar{A}_{t_{0}}+\bar{X}_{t_{0}}^{h}\right]\right|=\left|\log \left[\frac{\bar{A}_{t_{2}}+\bar{X}_{t_{2}}^{h}}{e^{\Delta} \bar{A}_{t_{0}}+\bar{X}_{t_{0}}^{h}}\right]\right| \\
& \leq \max \left[\frac{\bar{A}_{t_{2}}+\bar{X}_{t_{2}}^{h}}{e^{\Delta} \bar{A}_{t_{0}}+\bar{X}_{t_{0}}^{h}}-1, \frac{e^{\Delta} \bar{A}_{t_{0}}+\bar{X}_{t_{0}}^{h}}{\bar{A}_{t_{2}}+\bar{X}_{t_{2}}^{h}}-1\right] \\
& \leq \max \left[\frac{\left(\bar{A}_{t_{2}}-e^{\Delta} \bar{A}_{t_{0}}\right)+\left(\bar{X}_{t_{2}}^{h}-\bar{X}_{t_{0}}^{h}\right)}{e^{\Delta} \bar{A}_{t_{0}}+\bar{X}_{t_{0}}^{h}}, \frac{\left(e^{\Delta} \bar{A}_{t_{0}}-\bar{A}_{t_{2}}\right)+\left(\bar{X}_{t_{0}}^{h}-\bar{X}_{t_{2}}^{h}\right)}{\bar{A}_{t_{2}}+\bar{X}_{t_{2}}^{h}}\right] \\
& \leq \max \left[\frac{\left|\bar{A}_{t_{2}}-e^{\Delta} \bar{A}_{t_{0}}\right|+\left|\bar{X}_{t_{2}}^{h}-\bar{X}_{t_{0}}^{h}\right|}{e^{\Delta} \bar{A}_{t_{0}}}, \frac{\left|e^{\Delta} \bar{A}_{t_{0}}-\bar{A}_{t_{2}}\right|+\left|\bar{X}_{t_{0}}^{h}-\bar{X}_{t_{2}}^{h}\right|}{\bar{A}_{t_{2}}}\right] .
\end{aligned}
$$

For the first set of limits we assume $t_{1}=t_{2}$ and take them to $t_{0}$ simultaneously; for the second set of limits we first take $t_{1} \rightarrow t_{0}$ and then take $t_{2} \rightarrow t_{0}$. In either case, in both terms of the maximum, the numerator converges to zero in $L_{2 p}$ while the inverse of the denominator is bounded in $L_{2 p}$. By 
the Cauchy-Schwarz inequality, the terms themselves converge to zero in $L_{p}$, hence so does their maximum. The argument for a negative shock is analogous.

\section{A-3.4 Notation and proof structure}

Bounds. We use the notation that $K(\bar{t}, \Delta)$ refers to a family of random variables which are uniformly bounded independently of $\Delta$, in the limit as $t_{1} \rightarrow t_{0}$, when $t_{2}$ and $t_{3}$ are for appropriately chosen. Formally, we require that there exists a family of constants $K(p)$, such that given $p$, for any $\Delta$, we can find $t_{2}(\Delta, p)$ small enogh and $t_{3}(\Delta, p)$ large enough so that $\lim _{t_{1} \rightarrow t_{0}} \sup \left\|K\left(t_{1}, t_{2}(\Delta, p), t_{3}(\Delta, p)\right)\right\|_{p} \leq K(p)$. Different occurences of $K(\bar{t}, \Delta)$ may refer to different families of random variables and may have a different $K(p)$ values associated with them. For example, Lemma 10 implies that $\log \left[\bar{C}_{t_{2}}^{h}\left(+, t_{1}, \Delta\right)\right]=\log \left[e^{\Delta} \bar{A}_{t_{0}}+\bar{X}_{t_{0}}^{h}\right]+K(\bar{t}, \Delta)$.

Order of limits. The statement of Proposition 7 assumes that $n$ is large enough; this means that $\sigma_{A} / \sigma_{I}$ is small enough, while other parameters of the model, as described in Section 3.4, remain bounded. We first analyze the case in which $\sigma_{A}$ becomes small, and then establish the result when $\sigma_{I}$ becomes sufficiently large using a clock change.

\section{A-3.5 Long-term behavior}

Lemma 11 Suppose that $n$ is large enough and $\sigma_{A}$ is small enough. Then

$$
\left.\lim _{t_{3} \rightarrow \infty t_{1} \rightarrow t_{0}} \lim _{\frac{X_{t_{3}}}{h}\left(-, t_{1}, \Delta\right)}-\frac{\widetilde{X}_{t_{3}}^{h}}{\bar{A}_{t_{3}}\left(-, t_{1}, \Delta\right)}\right]=0 .
$$

The intuition for the Lemma is that $X_{t_{3}}$ is just a weighted sum of past $A_{s}$ values, with the weights for the distant past going to zero exponentially fast. Thus, if $A_{s}$ is multiplied by a constant after date $t_{0}$, then for $t_{3}$ large enough, most of the terms determining $X_{t_{3}}$ in this weighted sum will also be multiplied by that constant, and hence $X_{t_{3}} / A_{t_{3}}$ will be approximately the same as it would be on the no-shock path. The caveat is that the terms in the weighted average corresponding to the distant past, divided by current $A_{t_{3}}$, must not blow up. For this we need that $1 / A_{t_{3}}$ does not become big too quickly relative to the rate with which the weights on the past converge to zero. These weights go to zero at a given exponential rate, so if the variance of the $A_{t}$ process is not too big, we are fine.

Proof of Lemma 11. Suppressing in notation that we work with the "negative shock" processes, 
we have

$$
\begin{aligned}
& \lim _{t_{1} \rightarrow t_{0}} \frac{\bar{X}_{t_{3}}^{h}}{\bar{A}_{t_{3}}}=\lim _{t_{1} \rightarrow t_{0}} \frac{1}{\bar{A}_{t_{3}}} \int_{0}^{t_{3}} \xi^{* \prime}\left(t_{3}-s\right) \bar{A}_{s} d s+\left[\bar{x}-\xi^{*}\left(t_{3}\right)\right] \frac{\bar{A}_{0}}{\bar{A}_{t_{3}}} \\
& =\frac{1}{\bar{A}_{t_{3}}} \int_{t_{0}}^{t_{3}} \xi^{* \prime}\left(t_{3}-s\right) \widetilde{A}_{s} e^{-\Delta} d s+\frac{1}{\bar{A}_{t_{3}}} \int_{0}^{t_{0}} \xi^{* \prime}\left(t_{3}-s\right) \bar{A}_{s} d s+\left[\bar{x}-\xi^{*}\left(t_{3}\right)\right] \frac{\bar{A}_{0}}{\bar{A}_{t_{3}}} \\
& =e^{-\Delta} \frac{\tilde{X}_{t_{3}}^{h}}{\bar{A}_{t_{3}}}+\left(1-e^{-\Delta}\right) \frac{1}{\bar{A}_{t_{3}}}\left(\int_{0}^{t_{0}} \xi^{* \prime}\left(t_{3}-s\right) \bar{A}_{s} d s+\left[\bar{x}-\xi^{*}\left(t_{3}\right)\right] \bar{A}_{0}\right) \\
& =\frac{\widetilde{X}_{t_{3}}^{h}}{\widetilde{A}_{t_{3}}}+\left(1-e^{-\Delta}\right) \frac{1}{\widetilde{A}_{t_{3}} e^{-\Delta}}\left(\left[-\xi^{*}\left(t_{3}-s\right) \bar{A}_{s}\right]_{0}^{t_{0}}+\int_{0}^{t_{0}} \xi^{*}\left(t_{3}-s\right) d \bar{A}_{s}+\left[\bar{x}-\xi^{*}\left(t_{3}\right)\right] \bar{A}_{0}\right) \\
& =\frac{\widetilde{X}_{t_{3}}^{h}}{\widetilde{A}_{t_{3}}}+\left(e^{\Delta}-1\right) \frac{1}{\widetilde{A}_{t_{3}}}\left(-\xi^{*}\left(t_{3}-t_{0}\right) \bar{A}_{t_{0}}+\xi^{*}\left(t_{3}\right) \bar{A}_{0}+\int_{0}^{t_{0}} \xi^{*}\left(t_{3}-s\right) d \bar{A}_{s}+\left[\bar{x}-\xi^{*}\left(t_{3}\right)\right] \bar{A}_{0}\right) \\
& =\frac{\widetilde{X}_{t_{3}}^{h}}{\widetilde{A}_{t_{3}}}+\left(e^{\Delta}-1\right) \frac{1}{\widetilde{A}_{t_{3}}}\left(\int_{0}^{t_{0}} \xi^{*}\left(t_{3}-s\right) d \bar{A}_{s}+\bar{x}_{0}-\xi^{*}\left(t_{3}-t_{0}\right) \bar{A}_{t_{0}}\right) .
\end{aligned}
$$

Here the last term can be written as

$$
\left(e^{\Delta}-1\right) \frac{1}{\widetilde{A}_{t_{3}}}\left(\int_{0}^{t_{0}}\left(\xi^{*}\left(t_{3}-s\right)-\bar{x}\right) d \bar{A}_{s}+\bar{A}_{t_{0}}\left[\bar{x}-\xi^{*}\left(t_{3}-t_{0}\right)\right]\right) .
$$

Because, by Lemma $3,\left|\xi^{*}\left(t_{3}-s\right)-\bar{x}\right| \leq K_{1} e^{-K_{2}\left(t_{3}-s\right)}$ for some constants $K_{1}, K_{2}$ independent of $n$, it follows from Lemma 7 that, for $n$ large enough, the first term here converges to zero as $t_{3} \rightarrow \infty$. Also by Lemma 3 the second term converges to zero as $t_{3} \rightarrow \infty$.

Lemma 12 Suppose that $n$ is large enough and $\sigma_{A}$ is small enough. There exists a constant $K_{2}$ such that the following holds. For any $\Delta$, we can find $t_{2}$ and $t_{3}$ such that for all $t_{1}$ close enough to $t_{0}$,

$$
E\left[\log \bar{C}_{t_{3}}^{h}-\log \bar{C}_{t_{2}}^{h} \mid S\left(+, t_{1}, \Delta\right)\right]-E\left[\log \bar{C}_{t_{3}}^{h}-\log \bar{C}_{t_{2}}^{h} \mid S\left(-, t_{1}, \Delta\right)\right] \geq \Delta-K_{2}
$$

Proof. A key element of the proof is that we bound the left hand side for each realization, that is, without the expectations operator. However, because $S\left(+, t_{1}, \Delta\right)$ and $S\left(-, t_{1}, \Delta\right)$ are disjoint events, we can only do this using the "shock processess", which have the same distribution as the original processes conditioned on the shock events, but are defined on a common probability space.

Suppose first that the shock is positive. Supressing in notation that we work with the shock process, we have $\log \bar{C}_{t_{3}}^{h} \geq \log \bar{A}_{t_{3}}=\log \widetilde{A}_{t_{3}}+\Delta$. Moreover, by Lemma 10 , for $t_{2}$ close to $t_{0}$, we have

$$
\log \bar{C}_{t_{2}}^{h}=\log \left[e^{\Delta} \bar{A}_{t_{0}}+\bar{X}_{t_{0}}\right]+K(\bar{t}, \Delta)=\log \bar{A}_{t_{0}}+\Delta+K(\bar{t}, \Delta)
$$


where the second equality follows because, given that we condition on the history up to $t_{0}, \bar{X}_{t_{0}}^{h} / \bar{A}_{t_{0}}$ is a constant. We can now write, for a positive shock, that

$$
\log \bar{C}_{t_{3}}^{h}-\log \bar{C}_{t_{2}}^{h} \geq\left(\log \widetilde{A}_{t_{3}}+\Delta\right)-\left(\log \bar{A}_{t_{0}}+\Delta+K(\bar{t}, \Delta)\right)=\log \widetilde{A}_{t_{3}}-\log \bar{A}_{t_{0}}+K(\bar{t}, \Delta)
$$

Now suppose that the shock is negative. Then, using Lemma 10,

$\log \bar{C}_{t_{2}}^{h}=\log \left[e^{-\Delta} \bar{A}_{t_{0}}+\bar{X}_{t_{0}}\right]+K(\bar{t}, \Delta)=\log \bar{A}_{t_{0}}+\log \left[e^{-\Delta}+\bar{X}_{t_{0}} / \bar{A}_{t_{0}}\right]+K(\bar{t}, \Delta) \geq \log \bar{A}_{t_{0}}+K(\bar{t}, \Delta)$

because $\bar{X}_{t_{0}} / \bar{A}_{t_{0}}$ is a constant. Moreover, using the fact that $\log (1+z) \leq z$,

$$
\begin{aligned}
\log \bar{C}_{t_{3}}^{h} & =\log \left[\bar{A}_{t_{3}}+\bar{X}_{t_{3}}^{h}\right]=\log \bar{A}_{t_{3}}+\log \left[1+\bar{X}_{t_{3}}^{h} / \bar{A}_{t_{3}}\right] \\
& \leq \log \widetilde{A}_{t_{3}}-\Delta+\bar{X}_{t_{3}}^{h} / \bar{A}_{t_{3}}=\log \widetilde{A}_{t_{3}}-\Delta+\widetilde{X}_{t_{3}}^{h} / \widetilde{A}_{t_{3}}+K(\bar{t}, \Delta)
\end{aligned}
$$

where at the last step we used Lemma 11. It follows that for a negative shock

$$
\log \bar{C}_{t_{3}}^{h}-\log \bar{C}_{t_{2}}^{h} \leq \log \widetilde{A}_{t_{3}}-\Delta+\widetilde{X}_{t_{3}}^{h} / \widetilde{A}_{t_{3}}-\log \bar{A}_{t_{0}}+K(\bar{t}, \Delta)
$$

Combining the inequalities for the positive and the negative shocks yields, for the shock processes, the bound

$$
\begin{array}{r}
{\left[\log \bar{C}_{t_{3}}^{h}\left(+, t_{1}, \Delta\right)-\log \bar{C}_{t_{2}}^{h}\left(+, t_{1}, \Delta\right)\right]-\left[\log \bar{C}_{t_{3}}^{h}\left(-, t_{1}, \Delta\right)-\log \bar{C}_{t_{2}}^{h}\left(-, t_{1}, \Delta\right)\right] \geq} \\
\log \widetilde{A}_{t_{3}}-\log \bar{A}_{t_{0}}-\left(\log \widetilde{A}_{t_{3}}-\Delta+\widetilde{X}_{t_{3}}^{h} / \widetilde{A}_{t_{3}}-\log \bar{A}_{t_{0}}\right)+K(\bar{t}, \Delta) \\
=\Delta-\widetilde{X}_{t_{3}}^{h} / \widetilde{A}_{t_{3}}+K(\bar{t}, \Delta) .
\end{array}
$$

Finally,

$$
\frac{\widetilde{X}_{t_{3}}}{\widetilde{A}_{t_{3}}}=\frac{1}{\widetilde{A}_{t_{3}}} \int_{0}^{t_{3}} \xi^{*}\left(t_{3}-s\right) d \widetilde{A}_{s}+\bar{x} \frac{\widetilde{A}_{0}}{\widetilde{A}_{t_{3}}}=\bar{x}+\frac{1}{\widetilde{A}_{t_{3}}} \int_{0}^{t_{3}}\left[\xi^{*}\left(t_{3}-s\right)-\bar{x}\right] d \widetilde{A}_{s}
$$

and by Lemma 7 the last term is bounded in $L_{p}$ for all $t_{3}$. Thus the above difference is $\Delta$ plus a term bounded in $L_{p}$, and the claim of the Lemma follows. 


\section{A-3.6 Proofs of Propositions 6 and 7}

Proof of Proposition 6. (i) Taking expectations in the regression equation (12) conditional on the shock being positive respectively negative, and differencing, we obtain

$$
E\left[\log \bar{C}_{t_{1}}-\log \bar{C}_{t_{0}} \mid S\left(+, t_{1}, \Delta\right)\right]-E\left[\log \bar{C}_{t_{1}}-\log \bar{C}_{t_{0}} \mid S\left(-, t_{1}, \Delta\right)\right]=2 \beta_{1}\left(t_{1}, \Delta\right) \cdot \Delta
$$

which gives an expression for $\beta_{1}\left(t_{1}, \Delta\right)$. An analogous formula expresses $\beta_{1}^{h}\left(t_{1}, \Delta\right)$. Because $X_{t} / A_{t}$ is bounded from below by $L$ and from above by $U$, we have $\left|\log \left(\bar{C}_{t_{1}} / \bar{C}_{t_{0}}\right)-\log \left(\bar{A}_{t_{1}} / \bar{A}_{t_{0}}\right)\right| \leq$ $\log (1+U)-\log (1+L)=K_{1}$ and therefore

$$
\begin{aligned}
& E\left[\log \bar{C}_{t_{1}}-\log \bar{C}_{t_{0}} \mid S\left(+, t_{1}, \Delta\right)\right]-E\left[\log \bar{C}_{t_{1}}-\log \bar{C}_{t_{0}} \mid S\left(-, t_{1}, \Delta\right)\right] \\
\geq & E\left[\log \left(\bar{A}_{t_{1}} / \bar{A}_{t_{0}}\right) \mid S\left(+, t_{1}, \Delta\right)\right]-E\left[\log \left(\bar{A}_{t_{1}} / \bar{A}_{t_{0}}\right) \mid S\left(-, t_{1}, \Delta\right)\right]-2 K_{1}=2\left(\Delta-K_{1}\right) .
\end{aligned}
$$

Hence $\beta_{1}\left(t_{1}, \Delta\right) \geq 1-K_{1} / \Delta$.

(ii) Lemma 10 implies that for any positive $K_{2}$, we can choose $t_{1}$ close enogh to $t_{0}$ such that

$E\left[\log \bar{C}_{t_{1}} \mid S\left(+, t_{1}, \Delta\right)\right]-E\left[\log \bar{C}_{t_{1}} \mid S\left(-, t_{1}, \Delta\right)\right] \leq \log \left[e^{\Delta} \bar{A}_{t_{0}}+\bar{X}_{t_{0}}^{h}\right]-\log \left[e^{-\Delta} \bar{A}_{t_{0}}+\bar{X}_{t_{0}}^{h}\right]+K_{2}$.

The right-hand side can be bounded as

$\log \left[\frac{e^{\Delta} \bar{A}_{t_{0}}+\bar{X}_{t_{0}}^{h}}{e^{-\Delta} \bar{A}_{t_{0}}+\bar{X}_{t_{0}}^{h}}\right]=\log \left[\frac{e^{\Delta}+\bar{X}_{t_{0}}^{h} / \bar{A}_{t_{0}}}{e^{-\Delta}+\bar{X}_{t_{0}}^{h} / \bar{A}_{t_{0}}}\right] \leq \log \left[\frac{e^{\Delta}+\bar{X}_{t_{0}}^{h} / \bar{A}_{t_{0}}}{\bar{X}_{t_{0}}^{h} / \bar{A}_{t_{0}}}\right] \leq \Delta+\log \left[\frac{1+\bar{X}_{t_{0}}^{h} / \bar{A}_{t_{0}}}{\bar{X}_{t_{0}}^{h} / \bar{A}_{t_{0}}}\right]=\Delta+K_{3}$

where - given that we condition on the history up to $t_{0}-K_{3}$ is a constant. It then follows from (20) that, for a given $\Delta$, we can choose $t_{1}$ close enough to $t_{0}$ such that $\beta_{1}^{h}\left(t_{1}, \Delta\right)<1 / 2+\left(K_{2}+K_{3}\right) / \Delta$.

Proof of Proposition \%. (i) Taking expectations in (13) and differencing, we obtain

$$
E\left[\log \bar{C}_{t_{3}}-\log \bar{C}_{t_{2}} \mid S\left(+, t_{1}, \Delta\right)\right]-E\left[\log \bar{C}_{t_{3}}-\log \bar{C}_{t_{2}} \mid S\left(-, t_{1}, \Delta\right)\right]=2 \beta_{2}(\bar{t}, \Delta) \cdot \Delta
$$

which gives an expression for $\beta_{2}(\bar{t}, \Delta)$. An analogous formula expresses $\beta_{2}^{h}(\bar{t}, \Delta)$. Because $X_{t} / A_{t}$ is bounded from below by $L$ and from above by $U$, we have $\left|\log \left(\bar{C}_{t_{3}} / \bar{C}_{t_{2}}\right)-\log \left(\bar{A}_{t_{3}} / \bar{A}_{t_{2}}\right)\right| \leq$ 
$\log (1+U)-\log (1+L)=K_{1}$ and therefore

$$
\begin{aligned}
& E\left[\log \bar{C}_{t_{3}}-\log \bar{C}_{t_{2}} \mid S\left(+, t_{1}, \Delta\right)\right]-E\left[\log \bar{C}_{t_{3}}-\log \bar{C}_{t_{2}} \mid S\left(-, t_{1}, \Delta\right)\right] \\
\leq & E\left[\log \left(\bar{A}_{t_{3}} / \bar{A}_{t_{2}}\right) \mid S\left(+, t_{1}, \Delta\right)\right]-E\left[\log \left(\bar{A}_{t_{3}} / \bar{A}_{t_{2}}\right) \mid S\left(-, t_{1}, \Delta\right)\right]+2 K_{1}=2 K_{1} .
\end{aligned}
$$

Using (21) we obtain $\beta_{2}\left(t_{1}, t_{2}, t_{3}, \Delta\right) \leq K_{1} / \Delta$.

(ii) Using Lemma 12 we can find $t_{2}$ and $t_{3}$, and $t_{1}$ close enough to $t_{0}$, such that $\beta_{2}^{h}\left(t_{1}, t_{2}, t_{3}, \Delta\right) \geq$ $1-K_{2} / \Delta$. This gives the proof along a sequence $\Theta_{n}$ in which $\sigma_{A} \rightarrow 0$. Finally we discuss the case when as $n \rightarrow \infty$, we have $\sigma_{I} \rightarrow \infty$. The only step we need to verify is that Lemma 12 also holds for $n$ large enough. To show this, just like in the proof of our main result, we change the clock. Using the transformation introduced in Lemma 8, we let $\tau=1 / \sigma_{I}^{2}$ and slow down the model by rescaling deep parameters with $\tau$. In the habit representation of that "rescaled" model, for $n$ large enough Lemma 12 holds, because all the assumptions, in particular, the requirement that $\sigma_{A}$ is small enough, are satisfied. And because the habit representation of the model after the clock change is the same as changing the clock in the habit representation of the original model, it follows that - with appropriately unscaled values for $t_{2}$ and $t_{3}$-Lemma 12 also holds in the original model.

\section{A-4 Proofs for Sections 4.2 and 4.3}

Proof of Proposition 8. In $\bar{\Theta}^{*}$, agents in the interior of the band never adjust, hence $T_{*}\left(\widetilde{p} \mid x_{0}\right)=\infty$. For $n$ finite, agents does adjust eventually, but since the drift and variance of $y$ goes to zero, the expected time to adjustment approaches infinity. In the habit model, $x$ never changes, hence $T^{h, n}\left(\widetilde{p} \mid x_{0}\right)=\infty$.

Proof of Proposition 9. (i) Our first goal is to compute the value function of the habit agent. Let $\psi$ be defined so that the value function of the Merton consumption problem in the environment of the representative habit consumer, but without habit, is $\psi W^{1-\gamma} /(1-\gamma)$. By the envelope theorem, this Merton agent has consumption policy $c=\psi^{-1 / \gamma} W$. The surplus consumption of our habit agent is identical to the consumption of a Merton agent, because they solve the same maximization problem. Hence, if the habit consumer sets his initial surplus consumption to be $A_{0}$, the dollar cost of his lifetime surplus consumption expenditure is $A_{0} \psi^{1 / \gamma}$.

To proceed, we now evaluate the lifetime budget constraint of the habit consumer. Each dollar of consumption spending in a period also creates future expenditure in the form of increased habit. 
Suppose $1+B$ dollars is the present value of these future expenditures for a dollar of consumption spending today, where $B=0$ with no habits. Then $B$ must satisfy

$$
B=\int_{u=0}^{\infty} \theta(u) e^{-r u} d u \cdot(1+B)
$$

because each dollar of consumption creates $\theta(u)$ habit spending $u$ periods ahead, which has a total cost of $\theta(u)(1+B)$ in period $u$ dollars, which we must then discount back at the riskfree rate because these payments are certain. Solving yields

$$
B=\frac{1}{1-\int_{u=0}^{\infty} \theta(u) e^{-r u} d u}
$$

At any time $t$, our habit consumer also has pre-existing habit created by his past consumption. The dollar value of the expenditures generated is

$$
Z_{t}=(1+B) \cdot\left[\int_{s=0}^{t} C_{t-s} \int_{s}^{\infty} \theta(u) e^{-r(u-s)} d u d s+\int_{s=t}^{\infty} \theta_{0}(u) X_{0} e^{-r u} d u\right]
$$

where the term in parenthesis measures future consumption expenditures created by habits established before $t$, discounted back at the riskfree rate because these are certain; and the factor $1+B$ is included because each dollar of consumption spending has this total expenditure cost.

The consumer's lifetime budget constraint must then satisfy

$$
W_{t}=A_{t} \cdot \psi^{1 / \gamma}(1+B)+Z_{t}
$$

and his lifetime utility from surplus consumption, by the Merton value function, is simply $\psi^{1 / \gamma} A_{t}^{1-\gamma} /(1-\gamma)$. Combining these equations yields

$$
V_{t}^{\text {habit }}\left(W_{t}, X_{t}\right)=\frac{\psi}{1-\gamma}\left(\frac{W_{t}-Z_{t}}{1+B}\right)^{1-\gamma}
$$

The welfare of an individual commitment agent for a move-inducing negative wealth shock is proportional to $\left(w-\lambda_{1} x\right)^{1-\gamma} /(1-\gamma)$.

Now compare the welfare cost of shocks in the commitment and the habit economies. As wealth falls to zero, if $Z_{t}>0$ then the marginal utility of the habit agent will be driven to infinity even with a finite shock. In contrast, when $\lambda_{1}=0$, the marginal utility of the commitment agent only blows up when all his wealth is taken. It follows that for large finite shocks, $\Pi(q, b)$ is higher for 
the habit agent than in the commitment economy.

(ii) Begin with the commitment model. The agent in the limit economy never moves, and hence his value function is proportional to $(W-x / r)^{1-\gamma} /(1-\gamma)$. It follows that the coefficient of relative risk aversion $C R R A^{*}\left(W_{0}, x_{0}\right)=\gamma W_{0} /\left(W_{0}-x_{0} / r\right)$. Now consider an agent in economy $n$. Let $p_{0}$ denote the total dollar value at date zero of his total commitment expenditures on his current home. Given positive risk and growth, this agent does move eventually, implying $p_{0}<x_{0} / r$. One policy available to this consumer at any wealth $W$ is to maintain his spending and moving patterns on current commitments, and adjust spending proportionally on all other goods relative to the optimal policy with initial wealth $W_{0}$. Given that $\lambda_{1}=0$, this policy yields lifetime utility $V_{n}\left(W_{0}, x_{0}\right)\left(W-p_{0}\right)^{1-\gamma} /\left(W_{0}-p_{0}\right)^{1-\gamma}$. This is a lower bound for the agent's true value function, and the both equal $V_{n}\left(W_{0}, x_{0}\right)$ at $W_{0}$. It follows that the lower bound has higher curvature at $W_{0}$. As a result, $C R R A^{n}\left(W_{0}, x_{0}\right) \leq \gamma W_{0} /\left(W_{0}-p_{0}\right)$. Since $p_{0}<x_{0} / r$, we have $C R R A^{n}\left(W_{0}, x_{0}\right)<C R R A^{*}\left(W_{0}, x_{0}\right)$. Hence for $b$ small, the Arrow-Pratt approximation implies $\Pi^{n}(q, b)<\Pi^{*}(q, b)$ uniformly in $n$.

In the habit model, the value function in every economy is proportional to $(W-x / r)^{1-\gamma} /(1-\gamma)$, and hence $\Pi^{h, n}(q, b)=\Pi^{h *}(q, b)$.

\section{A-5 Simulations}

Solving the commitments model. In the simulations we use an ODE characterization of the optimal policy that builds on a similar characterization for the one-good model by Grossman and Laroque. To develop this ODE, we must study the Bellman equation of the commitment agent. By the mutual fund theorem the agent will combine the risky assets available to him in fixed proportions, effectively sharing his wealth between the mutual fund and the riskfree asset. Let $\pi_{r}$ and $\sigma_{r}$ denote the mean and standard deviation of the mutual fund's excess return. ${ }^{2}$ Denote the value function by $V(W, x)$, then the Bellman equation between adjustment dates is

$$
\rho V(W, x)=\max _{\alpha, \alpha}\left[\kappa \frac{a^{1-\gamma}}{1-\gamma}+\frac{x^{1-\gamma}}{1-\gamma}+V_{1}(W, x) E d W+\frac{1}{2} V_{11}(W, x) \operatorname{Var}(d W)\right]
$$

\footnotetext{
${ }^{2}$ In our setting we can use $\pi_{r}=\left[\left(\pi_{M} / \sigma_{M}\right)^{2}+\left(\pi_{M} / \sigma_{M}\right)^{2}\right] /\left[\pi_{M} / \sigma_{M}^{2}+\pi_{M} / \sigma_{M}^{2}\right]$ and $\sigma_{r}^{2}=$ $\pi_{r} /\left[\pi_{M} / \sigma_{M}^{2}+\pi_{M} / \sigma_{M}^{2}\right]$.
} 
Following Grossman and Laroque, let $y=W / X-\lambda_{1}$ and define $h(y)=x^{-1+\gamma} V(W, x)=$ $V(W / x, 1)$. Dividing through by $x^{1-\gamma}$ in the Bellman equation we obtain

$$
\rho h(y)=\max _{a, \alpha}\left[\kappa \frac{(a / x)^{1-\gamma}}{1-\gamma}+\frac{1}{1-\gamma}+h^{\prime}(y) E d y+\frac{1}{2} h^{\prime \prime}(y) \operatorname{Var}(d y)\right]
$$

and the budget constraint yields

$$
d y=\left(\left(y+\lambda_{1}\right)\left(r+\alpha \pi_{r}\right)-1-a / x\right) d t+\left(y+\lambda_{1}\right) \alpha \sigma_{r} d z .
$$

Maximizing in $\alpha$, the optimal portfolio satisfies

$$
\alpha\left(y+\lambda_{1}\right)=\frac{-h^{\prime}(y)}{h^{\prime \prime}(y)} \frac{\pi_{r}}{\sigma_{r}^{2}}
$$

and adjustable consumption is

$$
\frac{a}{x}=\left[\frac{h^{\prime}(y)}{\kappa}\right]^{-1 / \gamma}
$$

Substituting back into the Bellman equation we obtain

$$
\rho h(y)=h^{\prime}(y)^{1-1 / \gamma} \kappa^{1 / \gamma} \frac{\gamma}{1-\gamma}+\frac{1}{1-\gamma}+h^{\prime}(y)\left[\left(y+\lambda_{1}\right) r-1\right]-\frac{1}{2} \frac{h^{\prime}(y)^{2}}{h^{\prime \prime}(y)} \frac{\pi_{r}^{2}}{\sigma_{r}^{2}} .
$$

This is an ordinary differential equation for $h(y)$. To obtain boundary conditions, note that on an adjustment date the value function equals

$$
\begin{aligned}
\frac{V(W, x)}{x^{1-\gamma}} & =\frac{1}{x^{1-\gamma}} \max _{x^{\prime}} V\left(W-\lambda_{1} x-\lambda_{2} x^{\prime}, x^{\prime}\right) \\
& =\left(\frac{W-\lambda_{1} x}{x}\right)^{1-\gamma} \cdot \max _{x^{\prime}}\left(\frac{x^{\prime}}{W-\lambda_{1} x}\right)^{1-\gamma} \cdot V\left(\frac{W-\lambda_{1} x}{x^{\prime}}-\lambda_{2}, 1\right) \\
& =\left(\frac{W-\lambda_{1} x}{x}\right)^{1-\gamma} \cdot \max _{y}\left(y+\lambda_{1}+\lambda_{2}\right)^{-1+\gamma} h(y) .
\end{aligned}
$$

Define

$$
M=\max _{y}\left(y+\lambda_{1}+\lambda_{2}\right)^{-1+\gamma} h(y)
$$

then by the above reasoning, at the edges of the inaction band, denoted $y_{1}$ and $y_{2}$ we have

$$
h\left(y_{i}\right)=M y_{i}^{1-\gamma}
$$


moreover, smooth pasting implies

$$
h^{\prime}\left(y_{i}\right)=M(1-\gamma) y_{i}^{-\gamma}
$$

Finally, the target value of $y$ satisfies

$$
y^{*}=\arg \max \left(y+\lambda_{1}+\lambda_{2}\right)^{-1+\gamma} h(y) .
$$

To numerically solve the ODE subject to these conditions, we follow the approach outlined by Grossman and Laroque. We first pick some $M$, pick $y_{1}$, solve the ODE with initial conditions as given above. If there is no $y_{2}$ for which the boundary conditions are satisfied, then we start with a different $y_{1}$. If the boundary conditions do hold for some $y_{2}$, then we check if $M$ satisfies the equation above; if not, we start with a different $M$.

Simulating dynamics. We simulate the dynamics of an economy populated by a continuum of commitment agents using the partial differential equation of Proposition 3. We discretize the differential equation following the approach presented in Caballero (1993). We use this methodology to compute the steady state density $f^{*}$, to compute the impulse response (Definition 1 ), and to simulate dynamics along a sequence of aggregate shocks. We compute the matching consumption habit weights using Lemma 5 of this Appendix, and simulate the dynamics of the habit model using equation (10) of the main text.

\section{References to the Supplementary Appendix}

Ben-Ari, I. and Pinsky, R. (2007) "Spectral analysis of a family of second-order elliptic operators with nonlocal boundary condition indexed by a probability measure", Journal of Functional Analysis 251, 122-140.

Ben-Ari, I. and Pinsky, R. (2009). "Ergodic behavior of diffusions with random jumps from the boundary," Stochastic Processes and Applications 119, 864-881.

Grigorescu I. and Kang, M. (2002). "Brownian Motion on the Figure Eight", Journal of Theoretical Probability, 15(3), 817-844.

Karatzas, I. and Shreve, S. (2008). "Brownian Motion and Stochastic Calculus," Springer Verlag, 2nd edition.

Krylov, N. V. and Rozovski, B. L. (1977). "On the Cauchy Problem for Linear Stochastic Partial Differential Equations." Math. USSR Izvestija 12: 336-356. 
Krylov, N. V. and Rozovski, B. L. (1978). "On Conditional Distributions of Diffusion Processes." Math. USSR Izvestija 11: 1267-1284.

Lew, J. (1972). "On Linear Volterra Integral Equations of Convolution Type." Proceeedings of the American Mathematical Society 35(2): 450-456.

Revuz, D. and Yor, M. (1994). "Continuous Martingales and Brownian Motion," 2nd edition. In: Grundlehren der Mathematischen Wissenschaften [Fundamental Principles of Mathematical Sciences], vol. 293. Springer-Verlag, Berlin. 